\title{
Diffusion-Weighted Imaging Tractography-Based Parcellation of the Human Parietal Cortex and Comparison with Human and Macaque Resting-State Functional Connectivity
}

\author{
Rogier B. Mars, ${ }^{1,3}$ Saad Jbabdi, ${ }^{3}$ Jérôme Sallet, ${ }^{1}$ Jill X. 0'Reilly, ${ }^{3,4}$ Paula L. Croxson,,${ }^{1,4}$ Etienne Olivier, ${ }^{1,5}$ \\ MaryAnn P. Noonan, ${ }^{1}$ Caroline Bergmann, ${ }^{2}$ Anna S. Mitchell, ${ }^{1}$ Mark G. Baxter, ${ }^{4}$ Timothy E. J. Behrens, ${ }^{3}$ \\ Heidi Johansen-Berg, ${ }^{3}$ Valentina Tomassini, ${ }^{3}$ Karla L. Miller, ${ }^{3}$ and Matthew F. S. Rushworth ${ }^{1,3}$ \\ Departments of ${ }^{1}$ Experimental Psychology and ${ }^{2}$ Veterinary Services and ${ }^{3}$ Centre for Functional Magnetic Resonance Imaging of the Brain, University of \\ Oxford, Oxford OX1 2JD, United Kingdom, ${ }^{4}$ Glickenhaus Laboratory of Neuropsychology, Department of Neuroscience, Mount Sinai School of Medicine, \\ New York, New York 10029, and ${ }^{5}$ Institute of Neuroscience, Université Catholique de Louvain, B-1200 Brussels, Belgium
}

Despite the prominence of parietal activity in human neuroimaging investigations of sensorimotor and cognitive processes, there remains uncertainty about basic aspects of parietal cortical anatomical organization. Descriptions of human parietal cortex draw heavily on anatomical schemes developed in other primate species, but the validity of such comparisons has been questioned by claims that there are fundamental differences between the parietal cortex in humans and other primates. A scheme is presented for parcellation of human lateral parietal cortex into component regions on the basis of anatomical connectivity and the functional interactions of the resulting clusters with other brain regions. Anatomical connectivity was estimated using diffusion-weighted magnetic resonance image (MRI)-based tractography, and functional interactions were assessed by correlations in activity measured with functional MRI at rest. Resting-state functional connectivity was also assessed directly in the rhesus macaque lateral parietal cortex in an additional experiment, and the patterns found reflected known neuroanatomical connections. Crosscorrelation in the tractography-based connectivity patterns of parietal voxels reliably parcellated human lateral parietal cortex into 10 component clusters. The resting-state functional connectivity of human superior parietal and intraparietal clusters with frontal and extrastriate cortex suggested correspondences with areas in macaque superior and intraparietal sulcus. Functional connectivity patterns with parahippocampal cortex and premotor cortex again suggested fundamental correspondences between inferior parietal cortex in humans and macaques. In contrast, the human parietal cortex differs in the strength of its interactions between the central inferior parietal lobule region and the anterior prefrontal cortex.

\section{Introduction}

Research with both human and nonhuman primates shows that the parietal cortex is involved in numerous cognitive functions, from perception to control of action and higher-order processes, such as numerosity and memory. It is well established that parietal cortex consists of a mosaic of subregions, which are distinct both functionally and anatomically (Grefkes and Fink, 2005; Caminiti et al., 2010; Nelson et al., 2010). Our knowledge of the different subregions and their functional properties is primarily

\footnotetext{
Received Sept. 29, 2010; revised Dec. 15, 2010; accepted Jan. 11, 2011.

This work was supported by a Marie Curie Intra-European Fellowship within the 6th European Community Framework Programme (R.B.M.), the Wellcome Trust (M.P.N., M.G.B., H.J.-B.), the Medical Research Council (United Kingdom) (R.B.M., S.J., J.X.O., A.S.M., and M.F.S.R.), the Multiple Sclerosis Society (United Kingdom) (V.T.), the Italian Foundation for Multiple Sclerosis (V.T.), the Royal Academy of Engineering (K.L.M.), and the Economic and Social Research Council (United Kingdom) (K.L.M.). We thank Urs Schüffelgen for help with Caret analyses.

Correspondence should be addressed to Rogier B. Mars, Department of Experimental Psychology, University of 0xford, Tinbergen Building, 9 South Parks Road, 0xford 0X1 3UD, UK. E-mail: rogier.mars@psy.ox.ac.uk.

DOI:10.1523/JNEUROSCI.5102-10.2011

Copyright $\odot 2011$ the authors $\quad 0270-6474 / 11 / 314087-14 \$ 15.00 / 0$
}

based on the macaque model. However, the suitability of this model has been called into question (Passingham, 2009). The parietal cortex has expanded substantially in the human brain compared with other primates and even other hominids, which might have resulted in an increase or a rearrangement of its regions (Vanduffel et al., 2002b; Grefkes and Fink, 2005). Particular emphasis has been given to the possibility that human inferior parietal lobule is quite distinct to that of other species (Husain and Nachev, 2007).

The function of any brain area is constrained by its extrinsic connections. Indeed, patterns of anatomical connectivity can inform the segregation of functionally distinct areas (Passingham et al., 2002; Averbeck et al., 2009). Here, we aim to identify and characterize key component regions of human parietal cortex using a combination of non-invasive magnetic resonance imaging (MRI) techniques: probabilistic diffusion tractography and resting-state functional connectivity. The primary aim is to provide a parcellation of human parietal cortex that can inform noninvasive neuroimaging studies. An additional aim is to compare 
the parietal regions in the human brain with macaque parietal regions whose neurophysiology and connectional anatomy are well studied.

First, we determine the connectivity profiles of all human parietal voxels using diffusion-weighted magnetic resonance imaging (DW-MRI) and tractography and then parcellate the parietal cortex into distinct clusters based on the cross-correlations between these connectivity profiles (JohansenBerg et al., 2004; Anwander et al., 2007; Tomassini et al., 2007; Beckmann et al., 2009; Schubotz et al., 2010). Second, we examine the correlations between spontaneous activity in voxels in the parietal cortex and in other brain areas, a technique known as "resting-state functional connectivity" (O’Reilly et al., 2010; Xiang et al., 2010). The concept behind this resting-state functional MRI (fMRI) is that when the brain is "idle," i.e., not involved in any explicit task, correlations in slowly fluctuating spontaneous brain activity tend to reflect the intrinsic functional networks of the brain (Fox and Raichle, 2007). We focused on a limited set of eight areas known, in macaques, to project to circumscribed regions of parietal cortex. By comparing the correlations between the eight target areas with the tractography-based parietal clusters, we can characterize the distinct parietal subregions involved in various large-scale corticocortical interactions. Given the novelty of this approach, we first investigate resting-state interactions between parietal cortex and the same target areas in the macaque brain, to assess the degree to which the resting-state functional connectivity reflects known anatomical connections and thus to validate this method.

\section{Materials and Methods}

The research described in this paper consists of three parts. First, we parcellate the human parietal cortex on the basis of DW-MRI tractography. Second, we explore the potential of resting-state functional connectivity to describe interactions between the parietal cortex and other brain regions in both rhesus macaques and humans. Performing these analyses in macaques allow us to assess the degree to which functional connectivity reflects the prominent known anatomical connections in the same species. It is necessary to use macaques because the connections of macaque parietal cortex are well documented, but very little is known of the connections of human parietal cortex. Finally, having established the potential of resting-state functional interactions as indices of connectivity, we describe the patterns of functional interaction associated with each of the clusters found in the human data tractography-based parcellation of the human parietal cortex (Fig. 1A).

Diffusion-weighted data and tractography-based parcellation. Diffusionweighted images were acquired in eight healthy subjects (four female; age range, $20-36$ years; mean \pm SD age, $26.9 \pm 6.0$ years) on a $1.5 \mathrm{~T}$ Siemens Sonata MR scanner, with maximum gradient strength of $40 \mathrm{mT} \times \mathrm{m}^{-1}$. This number of participants has been established previously as sufficient for obtaining reliable parcellation results (Klein et al., 2007). All participants gave informed written consent in accordance with ethical approval from the local ethics committee. Participants lay supine in the scanner, and cushions were used to reduce head motion. Diffusion-weighted data were acquired using echo planar imaging $(72 \times 2 \mathrm{~mm}$ thick axial slices; matrix size, $128 \times 104$; field of view, $256 \times 208 \mathrm{~mm}^{2}$; giving a voxel size of $2 \times 2 \times 2 \mathrm{~mm}$ ). Diffusion weighting was isotropically distributed along 60 directions using a $b$ value of $1000 \mathrm{~s} \times \mathrm{mm}^{-2}$. For each set of
B

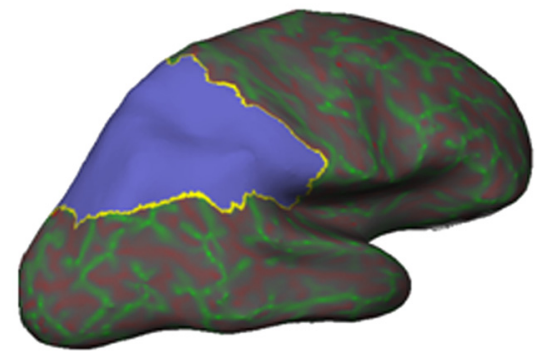

C

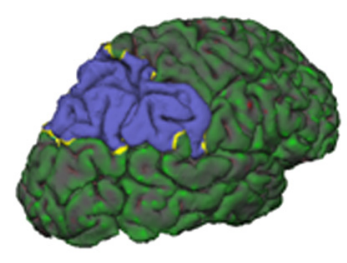

D

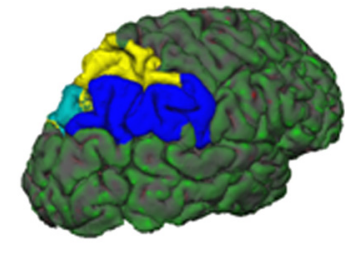
acquired throughout the acquisition. Three sets of diffusion-weighted data were acquired for subsequent averaging to improve the signal-tonoise ratio. The total scan time for the diffusion-weighted imaging protocol was $45 \mathrm{~min}$. A structural scan was acquired for each participant in the same session, using a T1-weighted three-dimensional fast, low-angle shot (3D FLASH) sequence [repetition time (TR), $12 \mathrm{~ms}$; echo time (TE), $5.65 \mathrm{~ms}$; flip angle, $19^{\circ}$; with elliptical sampling of $k$ space, giving voxel size of $1 \times 1 \times 1 \mathrm{~mm}$ ].

Analyses were performed using tools from FreeSurfer (Athinoula A. Martinos Center for Biomedical Imaging), FSL (for Functional MRI of the Brain Software Library) (Smith et al., 2004), Caret (Van Essen et al., 2001), and custom software written in Matlab (MathWorks). A parietal cortex region of interest (ROI) was drawn by hand in each participant's right hemisphere directly on the cortical surface model produced by FreeSurfer (Fig. $1 B-D$ ). In the absence of detailed cytoarchitectonic measurements, it is necessary to use macroscopic boundaries that can be reliably identified in all individuals as the boundaries of the ROI. The fundus of the inferior and superior postcentral sulci formed the rostral boundary of the ROI in its ventral and dorsal aspects, respectively. The ventral boundary was drawn between the ventral tip of the inferior postcentral sulcus and the conjunction of the horizontal and vertical branches of the Sylvian fissure, in the ventral supramarginal gyrus, and then to the conjunction of the ascending posterior segment of the superior temporal sulcus and horizontal posterior segment of the superior temporal sulcus. The boundary then followed the horizontal posterior segment of the superior temporal sulcus and continued to the nearest point on the intraparietal sulcus (IPS). The crown of the hemisphere constituted the medial boundary of the ROI. The point on the crown of the hemisphere nearest the parieto-occipital sulcus was the posterior boundary for the medial aspect of the mask, and it extended from there to the nearest point on the IPS. The lateral and medial banks of the full length of the entire IPS were included in the mask. The expanded fundus of the descending segment of the IPS, which is thought to correspond to extrastriate visual area V3A (Swisher et al., 2007), was excluded from the mask. Although there is individual variation in some aspects of the sulcal anatomy of the parietal cortex, these boundaries identified a similar location in the brains of all subjects after affine registration into Montreal Neurological Institute 
(MNI) space. It should be noted that, although the presence of both functional and anatomical differences between the hemispheres is well established in the human brain, especially in relation to language, the presence of hemispheric differences in the monkey brain is still a topic of debate (for review, see Passingham, 2008). Therefore, this study focuses on the right hemisphere, which is not dominant for language in humans.

DW-MRI data were preprocessed using tools from FDT (for Functional MRI of the Brain Diffusion Toolbox; part of FSL 4.1). Eddycurrent distortions were corrected using affine registration of all volumes to a target volume with no diffusion weighting. Voxelwise estimates of the fiber orientation distribution were calculated using Bedpostx, limited to estimating two fiber orientations at each voxel, because of the $b$ value and number of gradient orientations in the diffusion data (Behrens et al., 2007). For each participant, probabilistic tractography was run from vertices at the gray matter/white matter boundary surface within the parietal ROI to assess connectivity with every brain voxel (downsampled to $5 \mathrm{~mm}$ isotropic voxels), using a model accounting for multiple fiber orientations in each voxel (Behrens et al., 2007). Crucially, tractography was seeded from surface vertices (as opposed to voxels). From each seed vertex, the normal to the cortical surface was given by the FreeSurfer cortical model, and this information was used to track toward the brain instead of tracking in both directions as is usually done in voxel-based tractography. This approach dramatically reduces the risks of creating spurious sample tracts that cross gyral walls (for details, see www.fmrib.ox.ac.uk/fsl/fdt/fdt_surface.html). It also provides quantitative estimates of connectivity from every surface vertex of the parietal mask to every voxel in the rest of the brain.

A connectivity matrix between parietal vertices and each brain voxel was derived as described previously (Johansen-Berg et al., 2004) and used to generate a symmetric cross-correlation matrix of dimensions (number of seeds $\times$ number of seeds) in which the $(i, j)$ th element value is the correlation between the connectivity profile of seed $i$ and the connectivity profile of seed $j$. The rows or this cross-correlation matrix were then permuted using $k$-means segmentation for automated clustering to define different clusters. The goal of clustering the cross-correlation matrix is to group together regions that share the same connectivity with the rest of the brain. To increase the chances of obtaining continuous (nonscattered) clusters, we included a distance constraint (Tomassini et al., 2007). The parietal cortex contains a deep sulcus, the IPS, and the Euclidean distance between its banks is small although they are far apart in cortical distance. The flattening of the brain in FreeSurfer space, however, made it possible to implement the distance constraint along a geodesic that followed the cortical surface. The resulting clusters are then constrained to consist of vertices that are spatially contiguous, although the border between clusters is still guided by remote connectivity information.

The number of clusters in the $k$-means clustering must be set by the experimenter. To determine the optimal number of clusters resulting in consistency across participants, we used an iterative procedure (Beckmann et al., 2009). First, we looked for clusters to separate the parietal cortex mask into three separate partitions: a superior parietal lobule (SPL) mask, an inferior parietal lobule (IPL) mask, and a posterior IPS mask (Fig. 1 D). Following Beckmann et al. (2009), each of these masks was then parcellated into the maximum number of areas that led to a consistent parcellation in all eight participants. Each of the participant's individual clusters was then transformed from FreeSurfer space to the MNI template brain. The center of gravity of each cluster in MNI space and its overlap with the FSL implementation of the Harvard-Oxford probabilistic cortical atlas, an atlas based on manual definition of sulcal/ gyral landmarks, was then established.

Resting-state fMRI data acquisition and preprocessing. Human restingstate fMRI data and T1-weighted images were collected for a different group of 12 healthy volunteers (nine females; age range, 31-61 years; mean \pm SD age, $43.08 \pm 9.17$ years). This group of participants did not overlap with the group of participants in the diffusion-weighted imaging experiment. All participants gave informed written consent in accordance with ethical approval from the local ethics committee. Participants lay supine in a $1.5 \mathrm{~T}$ Siemens Sonata MR scanner. They were instructed to close their eyes and lie still. Cushions were used to reduce head motion.
Whole-brain blood oxygen level-dependent (BOLD) fMRI data was collected for $11 \mathrm{~min}$ from each participant, using the following parameters: 45 axial slices; in-plane resolution, $3 \times 3 \mathrm{~mm}$; slice thickness, $3 \mathrm{~mm}$; no slice gap; TR, $3400 \mathrm{~ms}$; TE, $41 \mathrm{~ms} ; 200$ volumes. A structural scan was acquired for each participant in the same session, using a T1-weighted 3D FLASH sequence (voxel size of $1 \times 1 \times 1 \mathrm{~mm}$ ).

Data were analyzed using tools from FSL (Smith et al., 2004) and SPM (for Statistical Parametric Mapping) (www.fil.ion.ucl.ac.uk/spm). The first six volumes of each functional dataset were discarded, after which the preprocessing was performed: motion correction, non-brain removal, spatial smoothing [using Gaussian $5 \mathrm{~mm}$ full-width at halfmaximum (FWHM) kernel], grand-mean intensity normalization of the entire four-dimensional dataset by a single multiplicative factor, and high-pass temporal filtering (Gaussian-weighted least-squares straight line fitting, with $\sigma=50.0 \mathrm{~s}$ ). Registration of functional images to the skull-stripped structural and MNI template was done using FLIRT (Jenkinson et al., 2002).

Macaque resting-state fMRI and anatomical scans were collected for 10 healthy macaques (Macaca mulatta; five females; age range, 3 years and 7 months to 4 years and 10 months; mean age, 4 years and 5 months; weight range, $4.34-8.53 \mathrm{~kg}$ ). Protocols for animal care, magnetic resonance imaging, and anesthesia were performed under authority of personal and project licenses in accordance with the United Kingdom Animals (Scientific Procedures) Act (1986). Anesthesia was induced using intramuscular injection of ketamine $(10 \mathrm{mg} / \mathrm{kg})$, xylazine $(0.125$ $0.25 \mathrm{mg} / \mathrm{kg})$, and midazolam $(0.1 \mathrm{mg} / \mathrm{kg})$. Macaques also received injections of atropine $(0.05 \mathrm{mg} / \mathrm{kg}$, i.m.), meloxicam $(0.2 \mathrm{mg} / \mathrm{kg}$, i.v. $)$, and ranitidine $(0.05 \mathrm{mg} / \mathrm{kg}$, i.v.). Local anesthetic (5\% lidocaine/prilocaine cream and $2.5 \%$ bupivacaine injected subcutaneously around the ears to block peripheral nerve stimulation) was also used at least $15 \mathrm{~min}$ before placing the macaque in the stereotaxic frame. The anesthetized animals were placed in an MRI-compatible stereotactic frame (Crist Instruments) in a sphinx position and placed in a horizontal $3 \mathrm{~T}$ MRI scanner with a full-size bore. Scanning commenced $\sim 2 \mathrm{~h}$ after induction, when the ketamine was unlikely still to be present in the system. Anesthesia was maintained using the lowest possible concentration of isoflurane to ensure that macaques were lightly anesthetized. The depth of anesthesia was assessed using physiological parameters (heart rate and blood pressure, as well as clinical checks before the scan for muscle relaxation). During the acquisition of the functional data, the inspired isoflurane concentration was in the range $1.0-1.8 \%$ (mean, $1.45 \%$ ), and the expired isoflurane concentration was in the range $0.9-1.7 \%$ (mean, $1.38 \%$ ). Isoflurane was selected for the scans as resting-state networks have been demonstrated previously to be present using this agent (Vincent et al., 2007). Macaques were maintained with intermittent positive pressure ventilation to ensure a constant respiration rate during the functional scan, and respiration rate, inspired and expired $\mathrm{CO}_{2}$, and inspired and expired isoflurane concentration were monitored and recorded using VitalMonitor software (Vetronic Services Ltd.). In addition to these parameters, core temperature and $\mathrm{SpO}_{2}$ were monitored throughout the scan. A four-channel phased-array coil was used for data acquisition $(\mathrm{H}$. Kolster, MRI Coil Laboratory, Laboratory for Neuro- and Psychophysiology, Katholieke Universiteit Leuven, Leuven, Belgium). Whole-brain BOLD fMRI data was collected for 53 min and $26 \mathrm{~s}$ from each animal, using the following parameters: 36 axial slices; in-plane resolution, $2 \times 2$ $\mathrm{mm}$; slice thickness, $2 \mathrm{~mm}$; no slice gap; TR, $2000 \mathrm{~ms}$; TE, $19 \mathrm{~ms} ; 1600$ volumes. A structural scan (three averages) was acquired for each macaque in the same session, using a T1-weighted magnetization-prepared rapid-acquisition gradient echo sequence (either $0.5 \times 0.5 \times 0.5$ or $0.5 \times$ $0.5 \times 1.0 \mathrm{~mm}$ voxel resolution)

The first six volumes of each functional dataset were discarded, and the following preprocessing was performed: non-brain removal, $0.1 \mathrm{~Hz}$ lowpass filtering to remove respiratory artifacts, motion correction, spatial smoothing (using Gaussian $3 \mathrm{~mm}$ FWHM kernel), grand-mean intensity normalization of the entire four-dimensional dataset by a single multiplicative factor, and high-pass temporal filtering (Gaussian-weighted least-squares straight line fitting, with $\sigma=50.0 \mathrm{~s}$ ). Registration of functional images to the skull-stripped structural and a macaque template was done using FLIRT (Jenkinson et al., 2002). 
Resting-state fMRI data analysis. To establish the functional connectivity of the parietal subregions yielded by the tractography-based parcellation, we created a number of target masks in MNI space from the probable homologs of brain areas that are known to be connected to the parietal cortex in the macaque. Functional interactions were determined with target areas in the ventral premotor cortex (PMv) centered at $[56,6,22]$ (Binkofski et al., 1999), dorsolateral prefrontal cortex (dlPFC) centered at [40, 40, 24] (Rajkowska and Goldman-Rakic, 1995), the parahippocampal gyrus ( $\mathrm{PH})$ centered at $[28,-34,-16]$ (Suzuki et al., 2005), the anterior prefrontal cortex (aPFC) centered at $[42,50,-2]$ (Mars et al., 2008; Boorman et al., 2009), V5/middle temporal area (MT) centered at $[44,-67,0]$ (Dumoulin et al., 2000), inferior dorsal premotor cortex (PMd) centered at MNI coordinates $[36,-2,50]$ (Hinkley et al., 2009), PMd centered at MNI coordinates [30, - 17, 64], (Amiez et al., 2006), and the frontal eye fields (FEF) centered at [24, $-6,44]$ (see Figs. 4, 9). All masks were of identical size $\left(6 \mathrm{~mm}^{3}\right)$, so as not to bias the analysis. Equivalent masks were created on the macaque template brain. The equivalent coordinates were determined by transferring the masks, using affine transformation, to the macaque template of McLaren et al. (2009), which uses the coordinates system of the atlas by Saleem and Logothetis (2006). The coordinates of the target areas were PMv [16, 24, 24], dlPFC [17, 37, 22], PH [11, 0, 7], aPFC [10, 44, 17], V5 [20,

$-1,18]$, inferior PMd $[16,22,29], \operatorname{PMd}[6,20,36]$, and FEF $[15,27,25]$ (see Figs. $3 A, 8 A$ ).

We used a voxelwise approach to map resting-state functional connectivity between the parietal cortex and characteristic time series associated with specific target regions. This analysis was performed in both human and macaques to (1) establish the reliability of the resting-state functional connectivity method as an index of known structural connectivity in the macaque brain and (2) allow a preliminary comparison of the functional interactions between the macaque and human brain. First, we calculated the major Eigen time series representing activity in each of the target region. The major Eigen time series is the single time series that best reflects coherent activity across the mask in that it represents the largest amount of variance across the set of voxels within the region. Then, we calculated the correlation between each voxel in the parietal cortex and the first Eigen time series of specific target areas for each participant. This was done using the seed-based correlation analysis tool, which is part of FSL (fsl_sbca) and is described by O'Reilly et al. (2010). Correlations between the parietal voxels and each target area were calculated separately. The average time series of the whole brain and the six movement parameters were used as confound regressors. The resulting correlation masks were then entered into a second-level group general linear model analysis. The resulting images were thresholded at $p<0.05$ for the parietal cortex region of interest (cf. O’Reilly et al., 2010).

Having established the reliability of the resting-state fMRI method and compared the patterns of correlation between the macaque and human brains, we then sought to formally compare the pattern of resting-state functional connectivity in parietal cortex associated with each of the target masks (PMv, dlPFC, PH, aPFC, V5, inferior PMd, PMd, and FEF) with the location of each of the parietal clusters established by tractography-based parcellation. Functional correlation maps of the target areas with the parietal cortex were overlaid with the thresholded clusters resulting from the tractography-based parcellation to reveal the resting-state connectivity fingerprint (Passingham et al., 2002), illustrating the interactions of each target area with the parietal clusters. For this analysis, the thresholded resting-state functional correlation map of each target area was binarized and multiplied by the thresholded (voxels belonging to a particular cluster in at least five participants), binarized tractography-based cluster masks. For each of the resulting overlay images resulting from this multiplication, the number of nonzero voxels indicates the strength of overlap between the area of functional interaction with a given target area and the tractography-based parietal cluster. The number of voxels in the overlay images were then first corrected for the size of each cluster and then normalized with respect to the total number of voxels correlating with the target mask across all clusters.

\section{Results}

\section{Inferior parietal lobule}

Tractography-based parcellation of the IPL subdivision (Fig. $1 D$, blue) identified five clusters, arranged consistently along the anteroposterior axis in all participants (Fig. $2 A, B$ ). The most anterior cluster (Fig. 2, red) with a center of gravity at $[49,-25,30]$ was most likely to be located in the parietal operculum according to Harvard-Oxford probabilistic atlas based and the Eickhoff et al. Anatomy Toolbox (Eickhoff et al., 2005). This region was similar in shape and location to the parietal opercular region (PFop) defined on cytoarchitectonic grounds by Caspers et al. $(2006,2008)$. This region, at least in the left hemisphere, is activated when people, but not macaques, observe tools being used, even if the macaques have experience of using the tools (Peeters et al., 2009). The tractography-based parcellation makes clear that this region is distinct from a more posterior region that is more commonly activated during grasping movements.

The two clusters (Fig. 2, blue and green) located directly posterior to PFop have centers of gravity at $[53,-32,44]$ and $[50$, $-44,43$ ] and were most likely to be located in anterior and posterior parts of the supramarginal gyrus. Again the areas correspond with ones defined on the basis of cytoarchitecture by Caspers et al. (2008). The more anterior of the two clusters (Fig. 2, 


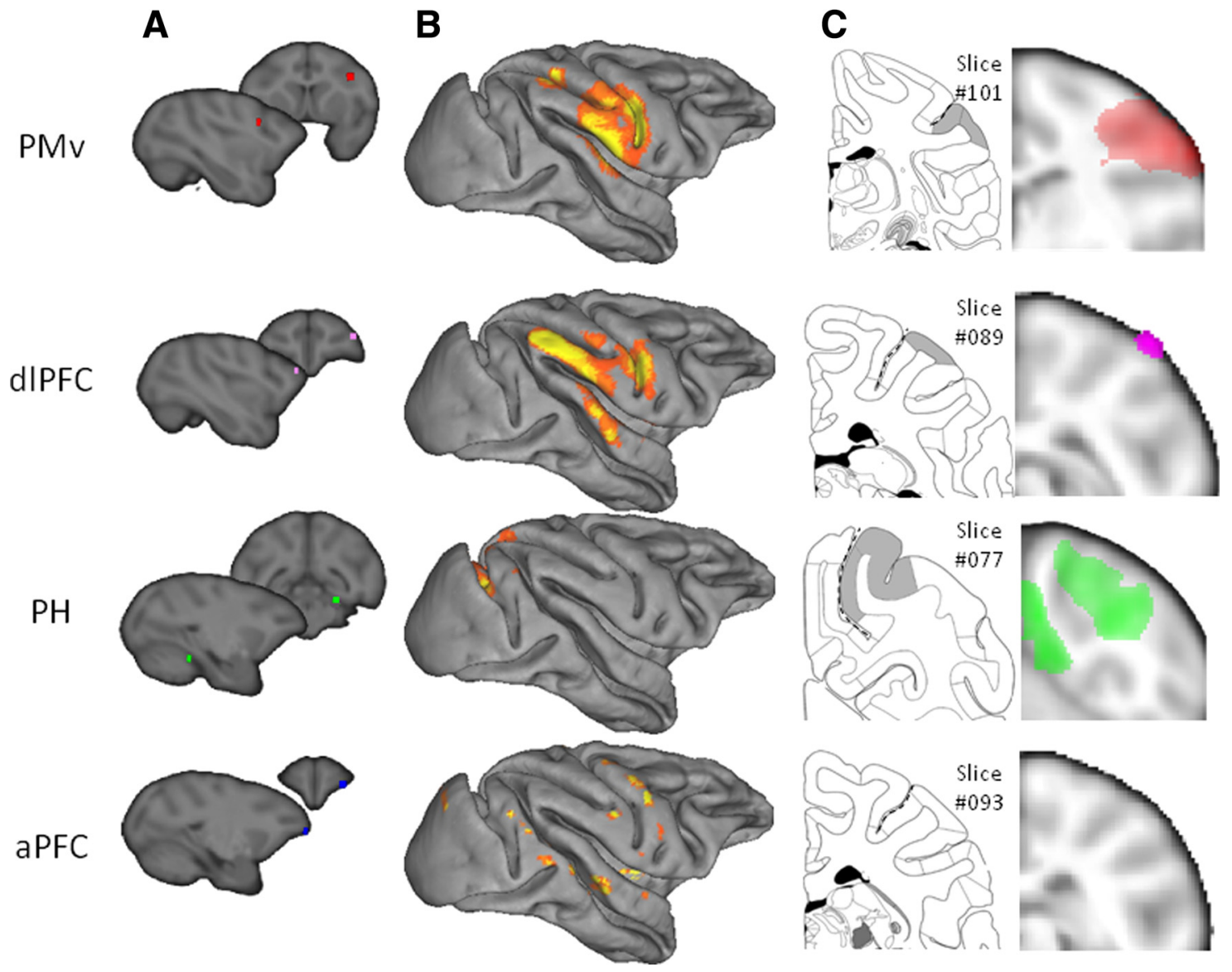

Figure 3. Macaque parietal resting-state functional interactions with PMv, dlPFC, PH, and aPFC. $A$, Target areas displayed on the template of McLaren et al. (2009), which uses the coordinate system of the atlas of Saleem and Logothetis (2006). B, Top 80\% of activity in the macaque parietal cortex correlating with the time course of activity in the target area, displayed on the Caret $F 99$ macaque template (Van Essen, 2002). C, Representative coronal slice of the macaque parietal cortex showing shaded areas that are hypothesized to show interactions with each respective target area, adopted from the atlas by Saleem and Logothetis (2006), and population resting-state fMRI results projected on the same slice (thresholded at $p<0.001$, except for PH thresholded at $p<$ 0.05). Dashed lines indicate intraparietal sulcus.

blue) resembles the conjunction of the PF and PFt regions defined by Caspers and colleagues. It is near the medial border of this region that activity is most often seen when human subjects grasp objects under visual guidance or manipulate them under visual guidance (Binkofski et al., 1999; Grefkes et al., 2002; Culham et al., 2003; Frey et al., 2005; Grol et al., 2007). As such, it might be a homolog of the monkey anterior intraparietal area (AIP). The more posterior of the two clusters (Fig. 2, green) resembles the PFm region defined by Caspers et al. (2008). Activity is recorded in this region under quite distinct circumstances. For example, when people make decisions, activity here increases in proportion with the evidence that the alternative choice would have been the better one to have taken and is especially prominent at the point that subjects switch between choices (Daw et al., 2006; Boorman et al., 2009).

The next most posterior cluster (Fig. 2, magenta), with a center of gravity of $[46,-55,45]$, corresponds most to the angular gyrus. Activity in this region is recorded when people redirect visuospatial attention from one location to another (Mort et al., 2003; Thiel et al., 2004). The most posterior cluster (Fig. 2, yellow/orange), with a center of gravity at $[37,-67,39]$, covers the most posterior and ventral parts of the angular gyrus and the most anterior part of the lateral occipital cortex. Activity in the most posterior IPL region has been noted when people successfully retrieve memories (Henson et al., 1999). Once again, the clusters correspond to cytoarchitectonic regions reported by Caspers et al. (2008). The anterior an- gular gyrus cluster resembles $\mathrm{PGa}$ and the posterior angular gyrus cluster resembles PGp.

We performed an explorative resting-state functional connectivity analysis looking at the correlation of IPL voxels with four target regions: $\mathrm{PMv}, \mathrm{PH}, \mathrm{dlPFC}$, and aPFC. In the macaque, PMv is known to be strongly connected to the anterior parts of the IPL, including areas AIP, PF, and PFG (Matelli et al., 1986; TanneGariepy et al., 2002). Resting-state functional connectivity of PMv with the IPL indeed was confined to the anterior IPL in macaques, suggesting that it reflects the known anatomical connections (Fig. 3). A similar pattern, with predominant interactions between anterior IPL and PMv, was seen in the human (Fig. $4 A$ ). In the macaque, the ventral part of dlPFC, where we placed our target, is also known to be interconnected with anterior IPL (Pandya and Yeterian, 1996), and indeed resting-state functional connectivity with dIPFC was most prominent in the same region of the IPL (Fig. 3). In contrast, PH is most strongly connected to posterior IPL regions in the macaque (Seltzer and Pandya, 1984; Suzuki and Amaral, 1994; Blatt et al., 2003). Once again, this pattern was also evident in the correlations between PH and IPL in both the macaque and the human (Fig. 4C). These results illustrate that resting-state functional connectivity reflects some of the most prominent anatomical connections found in macaque tracing studies and provides preliminary evidence of similarities between human and macaque IPL. 
A
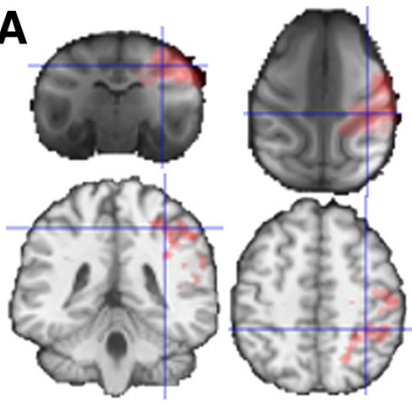

C

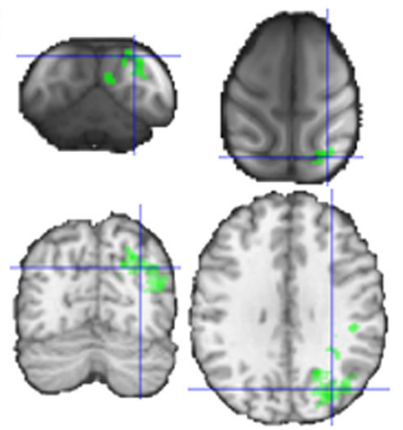

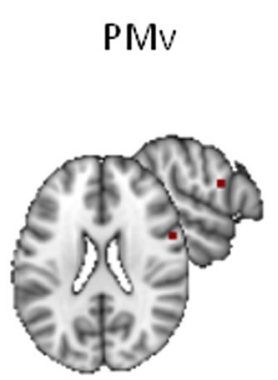

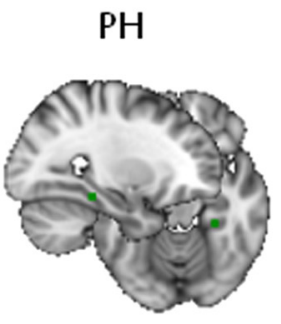

B

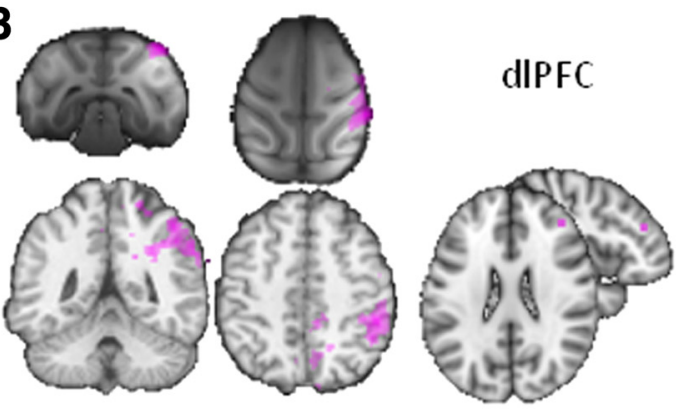

D

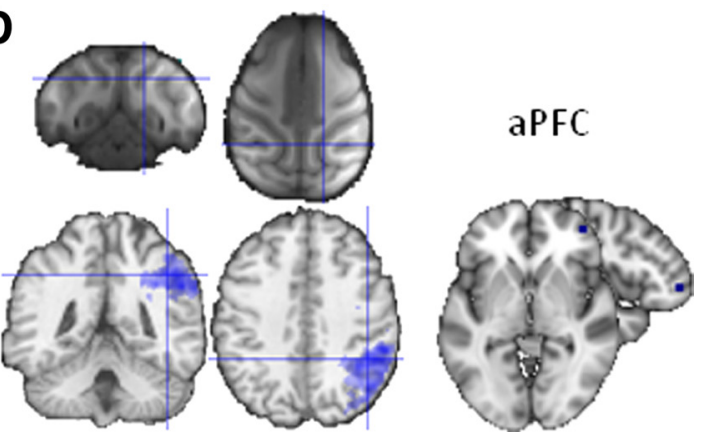

Figure 4. Human parietal resting-state functional interactions, target areas displayed on the MNI brain, and comparison with macaque resting-state fMRI results. $A$, Parietal activity correlating with PMv activity, showing anterior-IPL/PMv interactions in both humans and macaques. B, Parietal activity correlating with dIPFC activity, showing anterior-IPL/dIPFC interactions in both humans and macaques. C, Parietal activity correlating with PH activity, showing posterior-IPL/PH interactions in both humans and macaques. D, Parietal activity correlating with aPFC activity, showing mid-IPL/aPFC interactions in humans but not in macaques. All results thresholded at $p<0.05$.

It has been suggested that lateral parts of the parietal cortex (Denys et al., 2004a) or, perhaps more specifically, the IPL may have particularly expanded in humans compared with other primates (Simon et al., 2004; Husain and Nachev, 2007). Because there are a number of similarities between posterior IPL and anterior IPL in both humans and macaques, the central part of the IPL is the part of the IPL that is particularly likely to have expanded in humans compared with macaques (Rushworth et al., 2009). This region is engaged in complex cognitive tasks, such as numerical processing, task exploration, and task change ( $\mathrm{Si}$ mon et al., 2002; Daw et al., 2006). We therefore investigated the functional connectivity between the IPL and an area that is particularly extended in the homonids compared with the macaque brain, namely the aPFC (Semendeferi et al., 2001). In macaque, parietal cortex and aPFC are not strongly connected; there are no connections between parietal cortex the frontal polar area 10 (Petrides and Pandya, 2007), although there are some between the PFG region in the central part of the IPL and anterior parts of lateral prefrontal cortex (Rozzi et al., 2006). In humans, aPFC showed strong interactions with the central parts of the IPL (Fig. $4 D)$. In contrast, there was very little evidence for interactions between IPL and the aPFC regions used in the current investigation in the macaque even when the statistical threshold was reduced (Figs. 3, 4D).

These results indicate that IPL interactions with lateral PFC in the macaque are not as prominent as in humans or do not extend as far forward as in humans. Two additional analyses were performed to further investigate these results. First, to test whether the absence of interactions between aPFC and the central IPL in the macaque was simply a consequence of a difference in signal strength in aPFC in the two species, we looked for evidence of interactions between aPFC and the superior temporal cortex, a posterior brain area near to, but outside, the parietal cortex; connections between these two structures are known to exist in the macaque (Petrides and Pandya, 2007). Evidence for superior

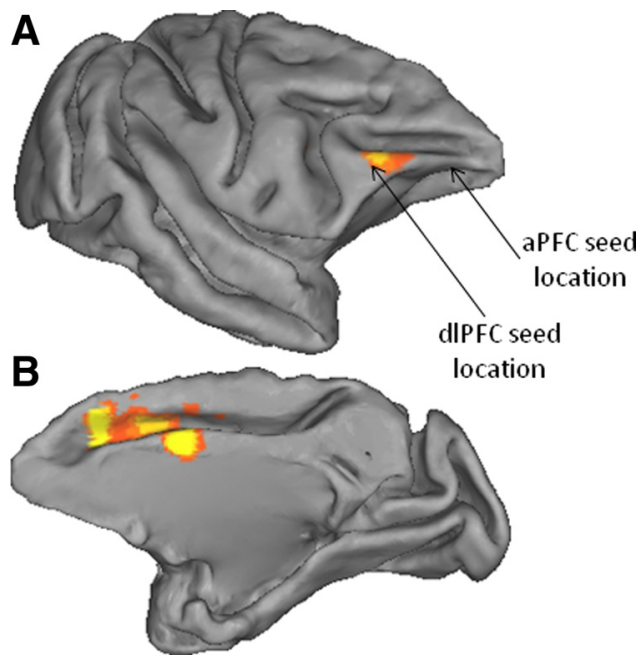

Figure 5. Macaque resting-state functional connectivity between IPL and prefrontal cortex. Top 80\% resting-state functional interactions between the mid-IPL area PFG and the lateral $(\boldsymbol{A})$ and medial ( $\boldsymbol{B}$ ) prefrontal cortex displayed on the Caret F99 macaque template (Van Essen, 2002).

temporal/aPFC interactions was apparent, indicating that the absence of IPL/aPFC interactions in the macaque is not attributable to lack of signal in the aPFC. Second, we further investigated the pattern of IPL/lateral PFC interactions in the macaque by placing a seed in macaque area PFG, in the medial IPL (coordinates [22, 5,29 ] in the atlas of Saleem and Logothetis, 2006) and establishing the location of functional interactions between this seed and the PFC. As shown in Figure 5A, lateral PFC interactions were evident near the location of our dlPFC seed but did not extend farther forward. In addition, prominent interactions were present between IPL and medial frontal cortex, particularly the cingulate motor areas (Fig. 5B). 


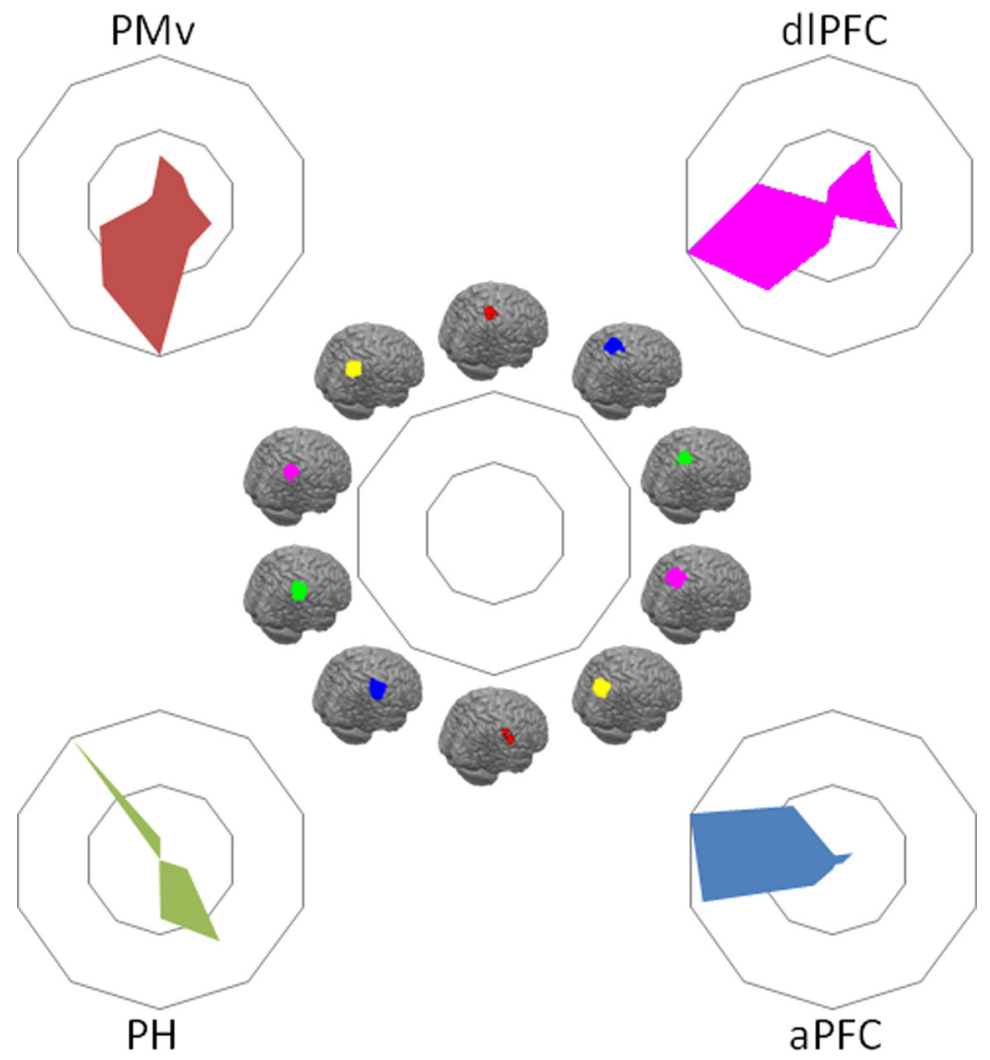

Figure 6. Comparison of tractography-based parcellation and human resting-state results for target areas hypothesized to interact strongly with the IPL. Connectivity fingerprints indicating the relative overlap between z-statistical images and the IPL clusters from the tractography-based parcellation shown in Figure 2 and the SPL parcellation shown in Figure 7, ranging from the most anterolateral SPL cluster (top of spider plot) clockwise to the most posterior SPL cluster and then from the most anterior IPL cluster (bottom of spider plot) to the most posterior IPL cluster. Connectivity fingerprints are from PMv (red), dIPFC (magenta), PH (green), and aPFC (blue). Data are normalized to reflect the relative contribution of each target area.

Finally, the human resting-state functional connectivity was formally compared with the tractography-based parcellation described above. Resting-state functional connectivity was determined between the five clusters reported in the parcellation and the PMv, dlPFC, PH, and aPFC target masks in second-level random-effects analyses, which allow inferences at the population level. These four target masks correlated with different IPL clusters (Fig. 6). The most anterior IPL clusters (particularly red and blue clusters in Fig. 2A) showed strong interactions with $\mathrm{PMv}$. This result is consistent with earlier diffusion-tractography work, showing that this region was most likely to connect to the ventral frontal cortex via the third branch of the superior longitudinal fasciculus (Croxson et al., 2005; Rushworth et al., 2006). It is also consistent with the fact that PMv, like the second most anterior IPL cluster, is active during reaching and grasping task (Tomassini et al., 2007). The second and third most anterior IPL regions (Fig. $2 \mathrm{~A}$, blue and green clusters) showed the strongest interactions with dlPFC. The most posterior IPL cluster (Fig. 2, yellow), covering the posterior part of the angular gyrus, showed interactions with $\mathrm{PH}$, again mimicking results obtained from diffusion tractography (Rushworth et al., 2006). As such, the results from the most anterior and most posterior portions of the IPL resemble those found in macaques. Resting-state correlation with the aPFC, which was much stronger in the human compared with the macaque, was strongest in the second and third most posterior clusters (Fig. $2 \mathrm{~A}$, magenta and green). Both this IPL region and $\mathrm{aPFC}$ are active when participants are implementing a decision to change their behavior based on growing evidence in favor of an alternative course of action (Fig. 2C) (Daw et al., 2006; Boorman et al., 2009).

\section{Superior parietal lobule and intraparietal sulcus}

The SPL and adjacent posterior IPS were consistently parcellated into four and two subdivisions, respectively. One cluster within the posterior IPS regions was located around [32, -76, 24] and overlapped with visual area V7 (Swisher et al., 2007) outside the parietal cortex. The remaining five regions, however, have consistently been designated parietal cortex, and so our analyses focused on them (Fig. 7). One area (Fig. 7, red) was located in an anterior position on the medial bank of the IPS with a center of gravity at [30, $-41,53]$. This area overlaps with the region identified as the ventral intraparietal area (VIP) on the basis of its activation by both visual and somatosensory stimulation adjacent to and on the face by Sereno and Huang (2006) and area 7PC on the basis of cytoarchitecture (Scheperjans et al., 2008b). This region is also close to an area identified by Bremmer et al. (2001) as involved in motion processing using visual, tactile, and auditory stimuli and subsequently suggested as the equivalent of macaque VIP. Adjacent to this area, but located more medially, was an area (Fig. 7, blue) with a center of gravity at $[12,-50$, $63]$ that overlaps with the cytoartchitectonically defined area 5L (Scheperjans et al., 2008b). Activity in this area is present during reaching movements and when reaching movements are adjusted when following a moving target (Diedrichsen et al., 2005).

Behind these two anterior SPL regions, we located a farther cluster in the more posterior part of the medial bank of the IPS (Fig. 7 , green), with a center of gravity at $[28,-55,55]$ that overlapped with the hIP3 region identified on the basis of cytoarchitecture by Scheperjans et al. (2008b). Activity is also seen in this area during visually guided reaching (Hinkley et al., 2009) and pointing (Astafiev et al., 2003), other visually guided hand movements (Grefkes et al., 2004) and when visuomotor contingencies are changed and updated (Rushworth et al., 2001). A retinopically organized area, IPS4, responding to visual stimulation has also been reported at this location (Swisher et al., 2007). A very similar region has been referred to as DIPSA (Vanduffel et al., 2002b; Denys et al., 2004a; Durand et al., 2009; Georgieva et al., 2009), aIPS (Astafiev et al., 2003), and SPL ant (Koyama et al., 2004), and a region referred to as ant IPs (Corbetta et al., 2000) lies slightly more ventrally but close by. Because activity in this region is particularly strong when hand and arm movements are made or hand and arm intentions are updated (Rushworth et al., 2001; Grefkes et al., 2004; Hinkley et al., 2009), it is reminiscent of the medial intraparietal region (MIP) in the macaque (Snyder et al., 1997). An alternative account, based partly on the shape sensitivity of the area, has suggested that it may resemble a posterior subregion of the AIP area found in the macaque (Orban et al., 2006; Durand et al., 2007, 2009). 
A fourth region was situated in a relatively medial and posterior position (Fig. 7 , magenta) with a center of gravity at [19, $-63,53]$. Its position therefore overlapped with the region that Scheperjans et al. (2008b) referred to as human 7A [and which bears no relation to the posterior IPL region in the macaque, which unfortunately has the same name (Cavada and Goldman-Rakic, 1989)]. The slightly more lateral position of the center of gravity of the area in the current study may be a consequence of not having included as much cortex on the medial wall in the present analysis. A retinotopic map, now often referred to as IPS3, has been reported in this area (Swisher et al., 2007) or close by (Sereno et al., 2001), and activity is more prominent in this region when the rules for guiding the allocation of visual attention are changed as opposed to the rules for guiding hand movements are changed (Rushworth et al., 2001). It has therefore been pointed out that this region bears some resemblance to the lateral intraparietal area (LIP) in the macaque. A very similar region has also been called DIPSM (Vanduffel et al., 2002b; Denys et al., 2004a; Durand et al., 2009; Georgieva et al., 2009), pIPS (Astafiev et al., 2003), and SPL post (Koyama et al., 2004).

Another cluster (Fig. 7, yellow) occupied a more ventral portion of the medial bank of the posterior IPS with a center of gravity at $[21,-78,43]$. Its position corresponded closely to that of the region referred to as POIPS (Vanduffel et al., 2002b; Denys et al., 2004a; Durand et al., 2009; Georgieva et al., 2009). The two most posterior retinopically mapped divisions of parietal cortex, IPS1 and IPS2, fall within this region (Silver et al., 2005; Swisher et al., 2007). Activity in this region is modulated by the allocation of visual attention and during delays while subjects memorize targets for intended eye movements (Schluppeck et al., 2005, 2006; Szczepanski et al., 2010). Again, similarities between IPS1 and IPS2 and the macaque LIP region have been noted. Attentional modulation of visual responses in anterior and dorsal parts of this cluster, together with the previously discussed cluster (Fig. 7, magenta), may possibly have been referred to as pos IPS, whereas attentional modulation of visual responses in more ventral and posterior parts, together with those in V7, may have been referred to as vIPS (Corbetta et al., 2000; Kincade et al., 2005). Despite the resemblances between response patterns reported in the human in the yellow and magenta clusters (Fig. 7) and the LIP region in the macaque, it is important to note that there are also important differences. For example, on the basis of a comparison of fMRI data from both species, it has been argued that the representation in monkey LIP is more biased to the contralateral hemifield than is the case in the human (Patel et al., 2010).

We used a resting-state functional connectivity analysis to determine the brain areas with which the SPL and IPS regions were interacting. An initial explorative resting-state functional connectivity analysis examined the correlation of parietal cortex voxels with four target regions in both macaque and human
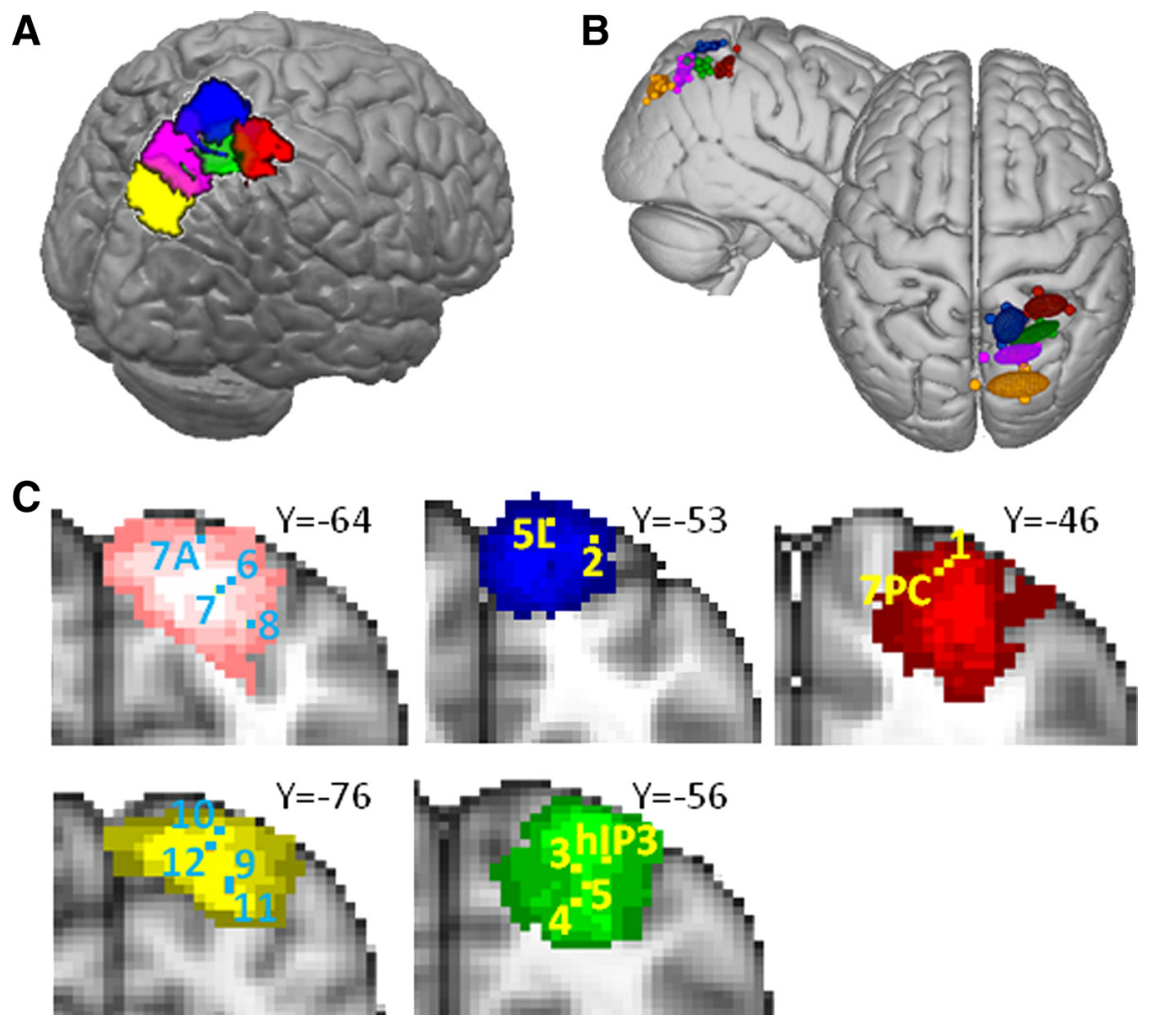

Figure 7. SPL/IPS tractography-based parcellation results. $\boldsymbol{A}$, Five clusters resulting from the tractography-based parcellation ov 2004); 6, IPS3 (Swisher et al., 2007); 7, LIP (Rushworth et al., 2001); 8, LIP (Sereno et al., 2001); 9, IPS1 (Swisher et al., 2007); 10, IPS2 (Swisher et al., 2007); 11, IPS1 (Silver et al., 2005); and 12, IPS2 (Silver et al., 2005).

brains: the extrastriate visual motion complex (V5/MT), PMd, an inferior PMd region, and FEF. The first region was at the approximate position of area V5/MT in the posterior superior temporal sulcus (Dubner and Zeki, 1971), which, within the macaque parietal cortex, is known to be interconnected with LIP and VIP (Blatt et al., 1990; Lewis and Van Essen, 2000). Within the macaque, parietal cortex functional connectivity with V5 is particularly strong in the posterior lateral bank of the IPS and fundus of the IPS at the approximate positions of LIP and VIP (Saleem and Logothetis, 2006) (Fig. 8). In the human subjects, however, prominent functional connectivity with V 5 was seen on the medial bank of the IPS (Fig. 9A). Two regions of correlation of activity in IPS were prominent: a small region in an anterior position near the first cluster [identified with 7PC and VIP (Fig. 7, red)] and a large one in a posterior position near the fourth and fifth clusters [identified with 7A and IPS3 (Fig. 7, magenta) and with IPS1/2 (Fig. 7, yellow)].

In the macaque, the pattern of functional connectivity with the inferior PMd region was complementary to the regions identified on the basis of their functional connectivity with V5/MT. Rather than predominantly implicating the LIP region on the lateral bank of the IPS, functional connectivity with inferior PMd was present in the medial bank of the IPS in the MIP region and adjacent SPL (Saleem and Logothetis, 2006). Such distinct patterns of functional interaction are reminiscent of the differences in structural connections of these regions; whereas LIP and VIP but not MIP are interconnected with V5, inferior PMd is more strongly interconnected with MIP than it is with LIP (Caminiti et 
A

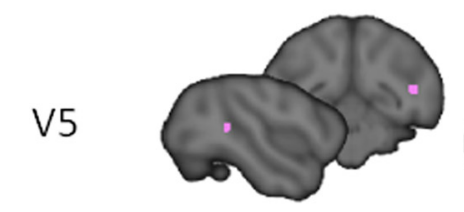

B
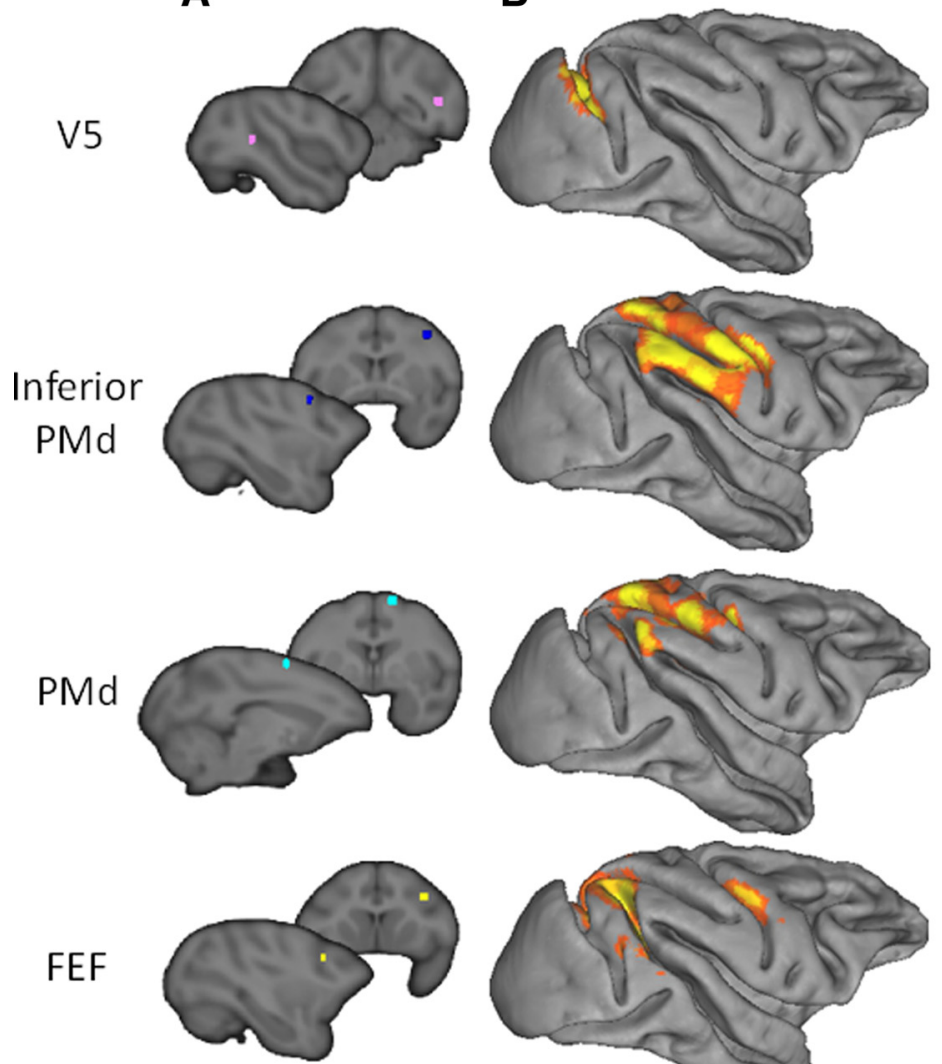

C
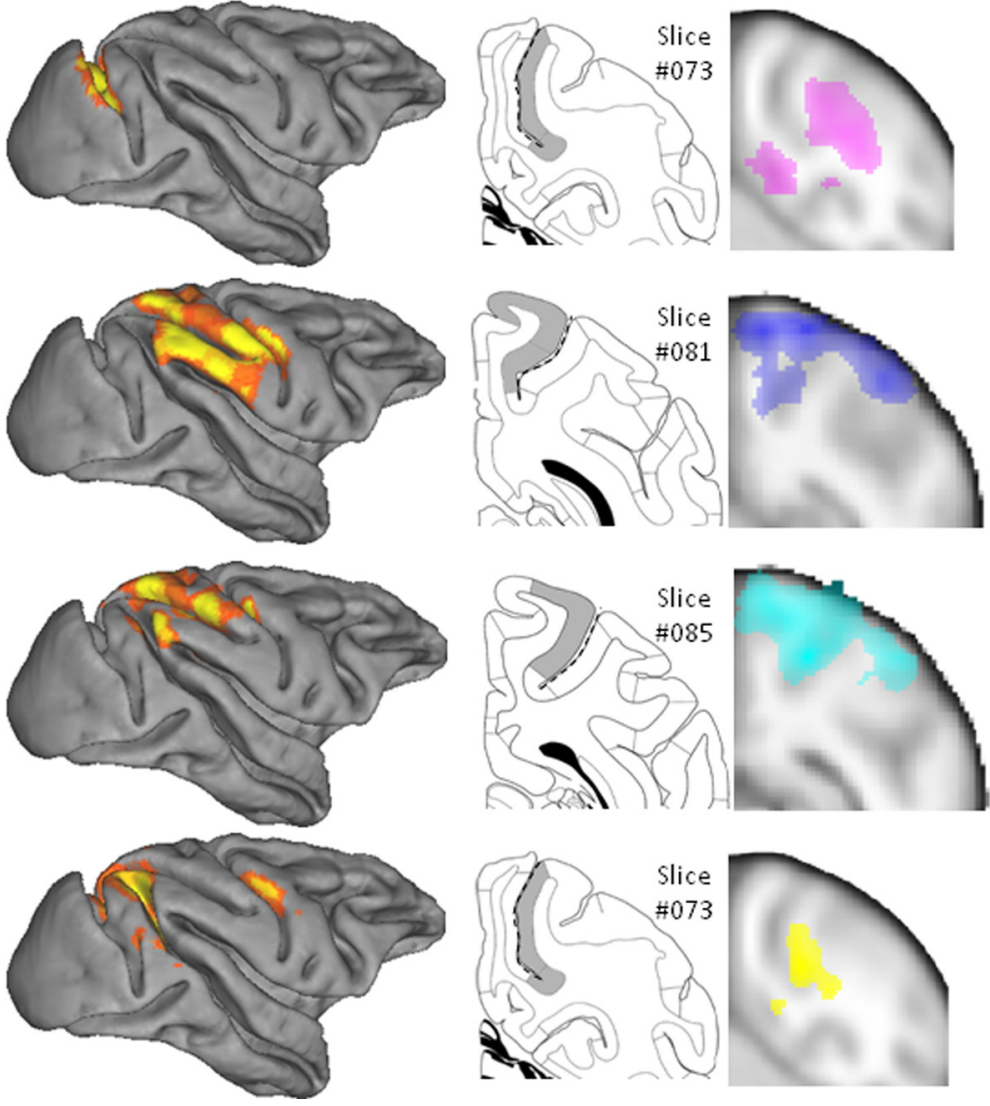

Figure 8. Macaque parietal resting-state functional interactions with V5, inferior PMd, PMd, and FEF. A, Target areas displayed on the template of McLaren et al. (2009), which uses the coordinate system of the atlas of Saleem and Logothetis (2006). B, Top 80\% of activity in the macaque parietal cortex correlating with the time course of activity in the target area, displayed on the Caret F99 macaque template (Van Essen, 2002). C, Representative coronal slice of the macaque parietal cortex showing shaded areas that are hypothesized to show interactions with each respective target area, adopted from the atlas by Saleem and Logothetis (2006), and population resting-state fMRI results projected on the same slice (thresholded at $p<0.001)$. Dashed lines indicate intraparietal sulcus.

A
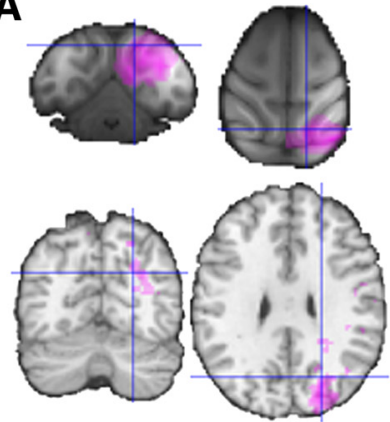

C
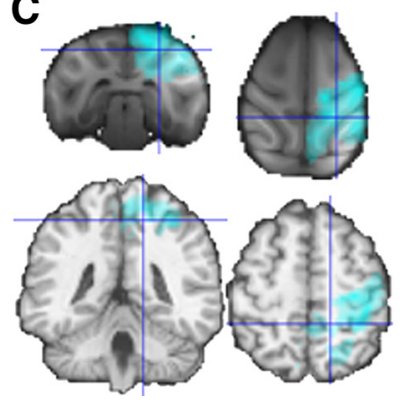

V5

B
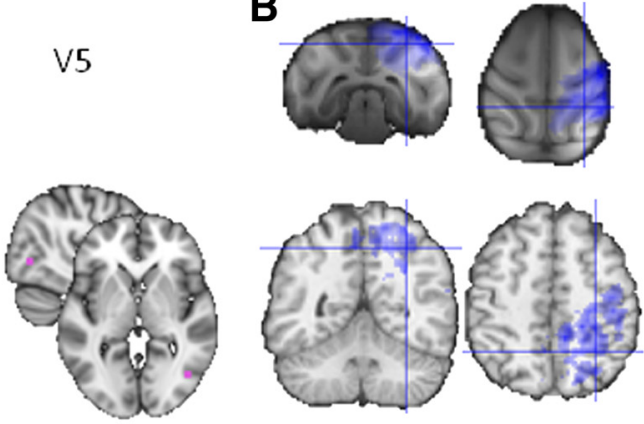

D
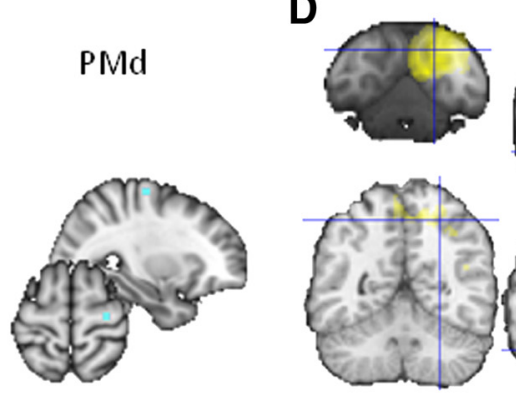

PMd

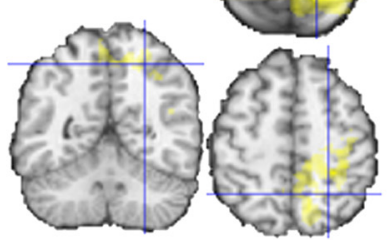

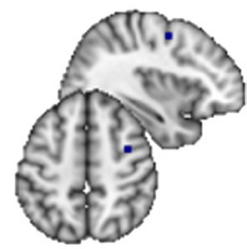

Inferior

PMd

FEF

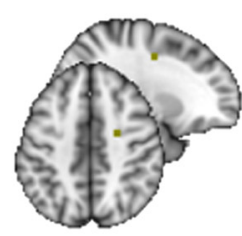

Figure 9. Human parietal resting-state functional interactions with V5 (A), inferior PMd (B), PMd (C), and FEF (D), target areas displayed on the MNI brain, and comparison with macaque resting-state fMRI results. All thresholded at $p<0.05$. 
al., 1996; Johnson et al., 1996; Matelli et al., 1998; Lewis and Van Essen, 2000). Similarly, there were apparent regional differences in the functional connectivity of V5/MT and the inferior PMd region in the human brain (Fig. 9).

We also compared the functional connectivity patterns associated with a more dorsal part of PMd and the FEF. In the macaque, there was evidence of functional interactions between PMd and a wide swath of parietal cortex that was centered on area 5 and MIP on the medial bank of the IPS but which extended into LIP and VIP on the lateral bank and fundus of the IPS (Saleem and Logothetis, 2006). In contrast, the functional interactions of FEF were most prominent in LIP. These results are comparable with the structural connections of PMd. PMd is known to be connected with parts of area 5 and extending into the adjacent parietal reach region including MIP (Caminiti et al., 1996; Johnson et al., 1996; Matelli et al., 1998; Lewis and Van Essen, 2000), but there is also evidence for interconnections between LIP and PMd (Lewis and Van Essen, 2000). In contrast, FEF is interconnected with LIP and VIP (Lewis and Van Essen, 2000). Within human parietal cortex functional connectivity with PMd was prominent with the rostral SPL and rostral medial bank of the IPS (Fig. 9C). Functional connectivity with the human FEF was focused on a large region in the anterior medial bank of the IPS [the area identified with 7PC and VIP (Fig. 7, red)] and a smaller region in the posterior medial bank of the IPS [the area identified with 7A and IPS3 (Fig. 7, magenta)].

As with the IPL, these results show that resting-state functional connectivity in SPL/IPS is found in areas that are known to be structurally interconnected and illustrate that patterns of functional connectivity are broadly comparable in the human and macaque. In the next stage of the analysis, the human restingstate functional connectivity was formally compared with the tractography-based parcellation described above. Resting-state functional connectivity was determined between the parietal clusters and V5, inferior PMd, PMd, and FEF (Fig. 10). Evidence for functional connectivity with V5/MT was found in the most posterior SPL cluster on the posterior medial bank of the IPS [identified with IPS1/2 (Fig. 7, yellow)], the adjacent fourth IPS region [identified with 7A and IPS3 (Fig. 7, magenta)], and the first IPS region [identified with 7PC and VIP (Fig. 7, red)]. Evidence for functional connectivity with PMd was found predominantly in three SPL/IPS clusters [identified with $5 \mathrm{~L}$ (Fig. 7, blue), hIP3/IPS4 (Fig. 7, green), and 7PC/VIP (Fig. 7, red)], with some limited connectivity also with the IPL (Wise et al., 1997). Evidence for functional connectivity within inferior PMd also included the same three SPL/IPS clusters, but interactions with the hIP3/IPS4 cluster (Fig. 7, green) were more prominent than had been the case with the more superior PMd region. The difference was particularly apparent at higher thresholds. This pattern resembles the pattern in the macaque in which PMd is interconnected with a number of SPL regions but a more inferior PMd region is particularly interconnected with MIP (Matelli et al., 1998).

Functional connectivity with the FEF (Fig. 10) was most prominent in the anterior lateral SPL/IPS region identified with 7PC and VIP (Fig. 7, red). Evidence for functional connectivity between the more posterior lateral SPL/IPS region identified with 7A and IPS3 (Fig. 7, magenta) and the SPL region identified with 5L (Fig. 7, blue) was also present. In addition, it should be noted that there is some uncertainty regarding the location of FEF in the human brain. In the macaque, FEF is located on the rostral bank of the arcuate sulcus. In the human brain, fMRI studies often activate a region more caudal than would be expected on the basis of macaque data, whereas stimulation studies position human FEF just anterior to the superior precentral sulcus (Amiez and Petrides, 2009). The target area used in the present study is located at the junction of the superior frontal sulcus and the precentral sulcus.

\section{Discussion}

The present study characterized the anatomical and functional organization of human lateral parietal cortex using non-invasive MRI techniques and compared it with the organization of the macaque parietal cortex. First, we used diffusion tractography to parcellate the human parietal cortex into 10 subregions. Then resting-state fMRI was used to examine the functional connectivity patterns of these subregions. Finally, comparing the restingstate fMRI data in humans and macaques revealed fundamental similarities in lateral parietal organization and demonstrated that 


\begin{tabular}{|c|c|c|c|c|}
\hline $\begin{array}{l}\text { DWI-defined } \\
\text { cluster }\end{array}$ & $\begin{array}{l}\text { Cytoarchitectonic } \\
\text { region }\end{array}$ & $\begin{array}{l}\text { Retinotopic } \\
\text { map }\end{array}$ & MGH/Leuven & $\begin{array}{l}\text { Other functional } \\
\text { nomenclature }\end{array}$ \\
\hline \multicolumn{5}{|l|}{ IPL } \\
\hline Red & PFop & & & \\
\hline Blue & $\mathrm{PF} / \mathrm{PFt} / \mathrm{hlP2}$ & & & AIP, hAIP \\
\hline Green & PFm & & & \\
\hline Magenta & PGa & & & \\
\hline Yellow & $P G p$ & & & \\
\hline \multicolumn{5}{|l|}{ SPL } \\
\hline Red & $7 P C$ & & & VIP \\
\hline Blue & $5 \mathrm{~L}$ & IPS5 & & \\
\hline Green & $\mathrm{hIP3}$ & IPS4 & DIPSA & $\begin{array}{l}\text { Human MIP, posterior AIP, } \\
\text { aIPS, SPL ant }\end{array}$ \\
\hline Magenta & $7 \mathrm{~A}$ & IPS3 & DIPSM & Pos IPS, SPLpost \\
\hline \multirow[t]{2}{*}{ Yellow } & & IPS2 & POIPS & Pos IPS \\
\hline & & IPS1 & POIPS & vIPS \\
\hline Not shown & & IPSO & VIPS, VIPS/V7 & vIPS, IPS/TOS \\
\hline
\end{tabular}

Diffusion-weighted imaging (DWI)-defined clusters are from the current study, and the colors listed refer to those shown in Figures 2 and 7. Cytoarchitectonic regions are based on the descriptions of Choi, Caspars, and Scheperjans and colleagues (Caspers et al., 2006, 2008; Choi et al., 2006; Scheperjans et al., 2008a,b). Retinotopic map designations are based on several studies (Schluppeck et al., 2005; Silver et al., 2005; Levy et al., 2007; Swisher et al., 2007 Konen and Kastner, 2008). The designations used by a group of researchers based in the Massachusetts General Hospital (MGH) and Katholieke Universiteit Leuven (Leuven) are listed in the fourth column (Vanduffel et al., 2002a; Denys et al., 2004b; Orban et al., 2006; Durand et al., 2007, 2009; Georgieva et al., 2009). Other designations based on the behavioral condition in which activations have been found are listed in the final column: a region referred to as AIP or hAIP region reported at the medial boundary of the second most anterior IPL cluster (Fig. 7, blue) (Binkofski et al., 1999; Grefkes et al., 2002: Culham et al.,2003; Frey et al., 2005; Grol et al.,2007):SPLant, SPL post, and IPS/TOS (Koyama et al., 2004); MIP (Rushworth et al., 2001; Grefkes et al., 2004); and pos IPS, vIPS, and alPS from Corbetta and colleagues (Corbetta et al., 2000; Astafiev et al., 2003; Kincade et al., 2005).

the resting-state functional connectivity of lateral parietal cortex reflected major known anatomical connections. There are fundamental similarities in lateral parietal organization in humans and macaques, but some features distinguished human parietal cortex. An anterior IPL region active during tool observation was identified (Fig. 2, red cluster), and a central IPL region was characterized by strong interactions with aPFC.

Diffusion-tractography approaches have identified component areas within the pre-supplementary motor area (pre-SMA)/ SMA region (Johansen-Berg et al., 2004), lateral premotor and ventrolateral prefrontal cortex (Anwander et al., 2007; Klein et al., 2007; Tomassini et al., 2007; Schubotz et al., 2010), and cingulate cortex (Beckmann et al., 2009), but this is the first time such a large expanse of cortex has been parcellated. Previous attempts to establish relationships between human and macaque parietal cortex have focused on retinotopic mapping, identification of similarities in functional activation profiles, and postmortem cytoarchitectonic analysis (Table 1). Because it has different strengths and weaknesses to these methods, the diffusion-tractography parcellation and functional connectivity approach provides independent and convergent evidence about the key component subdivisions of human parietal cortex.

The IPL parcellation yielded five clusters, and in most cases it was possible to identify their relationships with cytoartchitectonically defined areas PFop, PFm, PGa, and PGp (Caspers et al., 2008). One cluster (Fig. 2, blue), however, corresponded to several cytoarchitectonically defined areas, PF, PFt, and hIP2 (Caspers et al., 2006; Choi et al., 2006), which were presumably grouped together because of similarities in their connections. The differences in anatomical connections that gave rise to parcellation should mean that each IPL cluster differs in the access it has to the information represented in other cortical areas. Activity related to grasping, effecting strategy and response changes, attentional shifts, and memory retrieval, were preferentially asso- ciated with different clusters (Fig. 2C). Notably, the location of the most anterior IPL cluster was similar to that of activity recorded during tool use observation that has only been observed in human IPL (Peeters et al., 2009).

It has been claimed that human IPL may contain unique regions not found in other primates (Husain and Nachev, 2007; Peeters et al., 2009). Resting-state functional connectivity, however, in the posterior and anterior IPL, with PH, dlPFC, and PMv respectively, was similar in both humans and macaques (Fig. 4) and probably reflects similar anatomical connections in both species. In contrast, the human central IPL region was notable in that, unlike macaque central IPL, its activity was correlated with that of aPFC. It is in this region of the IPL that calculation- and language-related activity is found (Simon et al., 2002, 2004), and activity changes occur as people weigh up and change response strategy (Daw et al., 2006; Boorman et al., 2009). The aPFC region is close to the frontal pole in which activity has been related to the highest levels of cognitive control when "branching" between different subtasks is required (Koechlin et al., 1999). The two areas are often co-active (Daw et al., 2006; Boorman et al., 2009). There is evidence that the frontal pole is especially large in humans (Semendeferi et al., 2001) and that its increase in size is attributable to its lateral expansion, in hominoids, into the approximate region we found to be connected to mid-IPL. Consistent with our results, Petrides and Pandya (2007) have reported no connections between frontal polar area 10 and parietal cortex in the macaque, although Rozzi et al. (2006) note some between mid-IPL and anterior lateral prefrontal cortex. Brain differences emerge during speciation in a number of ways, including as a result of new connections invading an area or as specialized divisions of an area becoming spatially separate (Krubitzer, 1995, 2007), and such changes may underlie the present findings. The central IPL region is an important one to be considered in any attempt to account for the distinctive expansion of parietal cortex seen in humans even when comparison with other hominids (Bruner, 2010).

Parcellation of SPL and posterior IPS resulted in five clusters. Once again, it was possible to identify correspondences between the clusters and cytoarchitectonically defined regions (Fig. 7C), including 7PC, 5L, hIP3, and 7A (Scheperjans et al., 2008b). Retinotopic and other mapping procedures have been used to identify sensorimotor regions in IPS and SPL; the diffusion-tractography approach provides convergent evidence for the anatomical separation of many of these areas, including VIP (Bremmer et al., 2001; Sereno and Huang, 2006), also referred to as IPS5 (Konen and Kastner, 2008), IPS3, and IPS4 (Sereno et al., 2001; Swisher et al., 2007). One ventromedial posterior IPS cluster (Fig. 7, yellow), however, covered a region associated with two retinotopic maps, IPS1 and IPS2 (Silver et al., 2005), which have activity related to visuospatial attention and oculomotor intention (Schluppeck et al., 2005, 2006). IPS1, IPS2, and IPS3 have all been proposed as possible homologs of macaque LIP. The assignment of IPS1 and IPS2 to one cluster and the assignment of IPS3 to another cluster suggests that IPS1 and IPS2 share a number of connections and predicts that their functions may be especially similar. There is some evidence that both IPS1 and IPS2 are distinguished by the lateralization of their activity (Szczepanski et al., 2010). A modern, detailed study of the cytoartchitecture of this region has yet to be published, but again the diffusion-tractography results suggest that it may differ from the adjacent $7 \mathrm{~A}$ region that encompasses much of IPS3.

The patterns of functional connectivity associated with the different SPL/IPS parietal clusters clarify the relationship between the parietal cortex in the human and other primates and 
are suggestive of function. Macaque VIP is known to be connected with V5, PMd, PMv, and FEF (Luppino et al., 1999; Lewis and Van Essen, 2000), and the anterior lateral SPL/IPS putative 7PC/VIP/IPS5 region (Fig. 7, red) was found to be functionally connected with the same regions in the present study (Fig. 10). The anterior medial, putative $5 \mathrm{~L}$, region (Fig. 7, blue) interacted strongly with PMd. Connections between area 5 and PMd have also been documented in the macaque (Caminiti et al., 1996; Johnson et al., 1996; Matelli et al., 1998). The mid-lateral SPL/IPS region near the IPS4 map (Fig. 7, green) was found to be functionally connected to PMd, particularly inferior PMd, in a manner reminiscent of the connections known to exist between MIP and inferior PMd in macaque (Matelli et al., 1998). As in macaque MIP, such a pattern would be expected if the region were to be involved in the directional control of movement and complements evidence that a similar region is active during visually guided hand movements even in the absence of eye movements (Rushworth et al., 2001; Grefkes et al., 2004; Hinkley et al., 2009). The functional connectivity between the most posterior IPS region (Fig. 7, yellow), near IPS1 and IPS2 maps, and V5 suggested that it resembles the ventral division of macaque LIP (Lewis and Van Essen, 2000). There was evidence of functional connectivity between the fourth, posterior lateral SPL/IPS cluster (magenta, IPS3 location) and FEF, suggesting that it also bore resemblances to LIP. Human SPL/IPS organization differed from macaque parietal organization in that the areas engaged in functional interactions with both FEF and V5, important interactions for any area concerned with visuospatial attention, were most prominent on the medial rather than the lateral bank of the IPS (Grefkes and Fink, 2005).

In summary, we show the feasibility of using non-invasive MRI methods to establish the anatomical and functional subregions of the human parietal cortex and to compare these with the macaque. Although only tracer injection studies in animal models provide definitive evidence for existence of synaptic connections between brain regions, comparative MRI-based studies are important because they facilitate translation to the human brain. Similarly, although studies such as the present one cannot replace detailed cytoarchitectonic descriptions of brain areas, they indicate ways of exploiting such descriptions in in vivo investigations of human brain anatomy and function in both health and illness. The possibility that functional connectivity is mediated by polysynaptic pathways cannot be ruled out. Nevertheless, similar, previous functional connectivity studies have recently identified correspondences between medial parietal and adjacent posterior cingulate areas in humans and macaques (Margulies et al., 2009). The qualitative similarity in human and macaque IPL is notable, suggesting that the neurophysiology and anatomy of macaque IPL is likely to be an important guide for understanding human IPL (Caminiti et al., 2010). Nevertheless, within this framework, human specializations, especially interactions between mid-IPL and $\mathrm{aPFC}$, can be discerned.

\section{References}

Amiez C, Petrides M (2009) Anatomical organization of the eye fields in the human and non-human primate frontal cortex. Prog Neurobiol 89:220-230.

Amiez C, Kostopoulos P, Champod AS, Petrides M (2006) Local morphology predicts functional organization of the dorsal premotor region in the human brain. J Neurosci 26:2724-2731.

Anwander A, Tittgemeyer M, von Cramon DY, Friederici AD, Knösche TR (2007) Connectivity-based parcellation of Broca's area. Cereb Cortex 17:816-825.

Astafiev SV, Shulman GL, Stanley CM, Snyder AZ, Van Essen DC, Corbetta M
(2003) Functional organization of human intraparietal and frontal cortex for attending, looking, and pointing. J Neurosci 23:4689-4699.

Averbeck BB, Battaglia-Mayer A, Guglielmo C, Caminiti R (2009) Statistical Analysis of Parieto-Frontal Cognitive-Motor Networks. J Neurophysiol 102:1911-1920.

Beckmann M, Johansen-Berg H, Rushworth MF (2009) Connectivity-based parcellation of human cingulate cortex and its relation to functional specialization. J Neurosci 29:1175-1190.

Behrens TE, Berg HJ, Jbabdi S, Rushworth MF, Woolrich MW (2007) Probabilistic diffusion tractography with multiple fibre orientations: What can we gain? Neuroimage 34:144-155.

Binkofski F, Buccino G, Stephan KM, Rizzolatti G, Seitz RJ, Freund HJ (1999) A parieto-premotor network for object manipulation: evidence from neuroimaging. Exp Brain Res 128:210-213.

Blatt GJ, Andersen RA, Stoner GR (1990) Visual receptive field organization and cortico-cortical connections of the lateral intraparietal area (area LIP) in the macaque. J Comp Neurol 299:421-445.

Blatt GJ, Pandya DN, Rosene DL (2003) Parcellation of cortical Afferents to three distinct sectors in the parahippocampal gyrus of the rhesus monkey: An anatomical and neurophysiological study. J Comp Neurol 466:161-179.

Boorman ED, Behrens TE, Woolrich MW, Rushworth MF (2009) How green is the grass on the other side? Frontopolar cortex and the evidence in favor of alternative courses of action. Neuron 62:733-743.

Bremmer F, Schlack A, Shah NJ, Zafiris O, Kubischik M, Hoffmann K, Zilles K, Fink GR (2001) Polymodal motion processing in posterior parietal and premotor cortex: A human fMRI study strongly implies equivalencies between humans and monkeys. Neuron 29:287-296.

Bruner E (2010) Morphological differences in the parietal lobes within the human genus. A neurofunctional perspective. Curr Anthropol 51:S77-S88.

Caminiti R, Ferraina S, Johnson PB (1996) The sources of visual information to the primate frontal lobe: A novel role for the superior parietal lobule. Cereb Cortex 6:319-328.

Caminiti R, Chafee MV, Battaglia-Mayer A, Averbeck BB, Crowe DA, Georgopoulos AP (2010) Understanding the parietal lobe syndrome from a neurophysiological and evolutionary perspective. Eur J Neurosci 31:2320-2340.

Caspers S, Geyer S, Schleicher A, Mohlberg H, Amunts K, Zilles K (2006) The human inferior parietal cortex: Cytoarchitectonic parcellation and interindividual variability. Neuroimage 33:430-448.

Caspers S, Eickhoff SB, Geyer S, Scheperjans F, Mohlberg H, Zilles K, Amunts K (2008) The human inferior parietal lobule in stereotaxic space. Brain Struct Funct 212:481-495.

Cavada C, Goldman-Rakic PS (1989) Posterior parietal cortex in rhesus monkey: II. Evidence for segregated corticocortical networks linking sensory and limbic areas with the frontal lobe. J Comp Neurol 287:422-445.

Choi HJ, Zilles K, Mohlberg H, Schleicher A, Fink GR, Armstrong E, Amunts K (2006) Cytoarchitectonic identification and probabilistic mapping of two distinct areas within the anterior ventral bank of the human intraparietal sulcus. J Comp Neurol 495:53-69.

Corbetta M, Kincade JM, Ollinger JM, McAvoy MP, Shulman GL (2000) Voluntary orienting is dissociated from target detection in human posterior parietal cortex. Nat Neurosci [Erratum (2000) 3:521] 3:292-297.

Croxson PL, Johansen-Berg H, Behrens TE, Robson MD, Pinsk MA, Gross CG, Richter W, Richter MC, Kastner S, Rushworth MF (2005) Quantitative investigation of connections of the prefrontal cortex in the human and macaque using probabilistic diffusion tractography. J Neurosci 25:8854-8866.

Culham JC, Danckert SL, DeSouza JF, Gati JS, Menon RS, Goodale MA (2003) Visually guided grasping produces fMRI activation in dorsal but not ventral stream brain areas. Exp Brain Res 153:180-189.

Daw ND, O’Doherty JP, Dayan P, Seymour B, Dolan RJ (2006) Cortical substrates for exploratory decisions in humans. Nature 441:876-879.

Denys K, Vanduffel W, Fize D, Nelissen K, Peuskens H, Van Essen D, Orban GA (2004a) The processing of visual shape in the cerebral cortex of human and nonhuman primates: A functional magnetic resonance imaging study. J Neurosci 24:2551-2565.

Denys K, Vanduffel W, Fize D, Nelissen K, Sawamura H, Georgieva S, Vogels $\mathrm{R}$, Van Essen D, Orban GA (2004b) Visual activation in prefrontal cortex is stronger in monkeys than in humans. J Cogn Neurosci 16:1505-1516. 
Diedrichsen J, Hashambhoy Y, Rane T, Shadmehr R (2005) Neural correlates of reach errors. J Neurosci 25:9919-9931.

Dubner R, Zeki SM (1971) Response properties and receptive fields of cells in an anatomically defined region of superior temporal sulcus in monkey. Brain Res 35:528-532.

Dumoulin SO, Bittar RG, Kabani NJ, Baker CL Jr, Le Goualher G, Bruce Pike G, Evans AC (2000) A new anatomical landmark for reliable identification of human area V5/MT: a quantitative analysis of sulcal patterning. Cereb Cortex 10:454-463.

Durand JB, Nelissen K, Joly O, Wardak C, Todd JT, Norman JF, Janssen P, Vanduffel W, Orban GA (2007) Anterior regions of monkey parietal cortex process visual 3D shape. Neuron 55:493-505.

Durand JB, Peeters R, Norman JF, Todd JT, Orban GA (2009) Parietal regions processing visual 3D shape extracted from disparity. Neuroimage 46:1114-1126.

Eickhoff SB, Stephan KE, Mohlberg H, Grefkes C, Fink GR, Amunts K, Zilles K (2005) A new SPM toolbox for combining probabilistic cytoarchitectonic maps and functional imaging data. Neuroimage 25:1325-1335.

Fox MD, Raichle ME (2007) Spontaneous fluctuations in brain activity observed with functional magnetic resonance imaging. Nat Rev Neurosci 8:700-711.

Frey SH, Vinton D, Norlund R, Grafton ST (2005) Cortical topography of human anterior intraparietal cortex active during visually guided grasping. Cogn Brain Res 23:397-405.

Georgieva S, Peeters R, Kolster H, Todd JT, Orban GA (2009) The processing of three-dimensional shape from disparity in the human brain. J Neurosci 29:727-742.

Grefkes C, Fink GR (2005) The functional organization of the intraparietal sulcus in humans and monkeys. J Anat 207:3-17.

Grefkes C, Weiss PH, Zilles K, Fink GR (2002) Crossmodal processing of object features in human anterior intraparietal cortex: an fMRI study implies equivalencies between humans and monkeys. Neuron 35:173-184.

Grefkes C, Ritzl A, Zilles K, Fink GR (2004) Human medial intraparietal cortex subserves visuomotor coordinate transformation. Neuroimage 23:1494-1506.

Grol MJ, Majdandzić J, Stephan KE, Verhagen L, Dijkerman HC, Bekkering H, Verstraten FA, Toni I (2007) Parieto-frontal connectivity during visually guided grasping. J Neurosci 27:11877-11887.

Henson RN, Rugg MD, Shallice T, Josephs O, Dolan RJ (1999) Recollection and familiarity in recognition memory: An event-related functional magnetic resonance imaging study. J Neurosci 19:3962-3972.

Hinkley LB, Krubitzer LA, Padberg J, Disbrow EA (2009) Visual-manual exploration and posterior parietal cortex in humans. J Neurophysiol 102:3433-3446.

Husain M, Nachev P (2007) Space and the parietal cortex. Trends Cogn Sci 11:30-36.

Jenkinson M, Bannister P, Brady M, Smith S (2002) Improved optimization for the robust and accurate linear registration and motion correction of brain images. Neuroimage 17:825-841.

Johansen-Berg H, Behrens TE, Robson MD, Drobnjak I, Rushworth MF, Brady JM, Smith SM, Higham DJ, Matthews PM (2004) Changes in connectivity profiles define functionally distinct regions in human medial frontal cortex. Proc Natl Acad Sci U S A 101:13335-13340.

Johnson PB, Ferraina S, Bianchi L, Caminiti R (1996) Cortical networks for visual reaching: Physiological and anatomical organization of frontal and parietal lobe arm regions. Cereb Cortex 6:102-119.

Kincade JM, Abrams RA, Astafiev SV, Shulman GL, Corbetta M (2005) An event-related functional magnetic resonance imaging study of voluntary and stimulus-driven orienting of attention. J Neurosci 25:4593-4604.

Klein JC, Behrens TE, Robson MD, Mackay CE, Higham DJ, Johansen-Berg H (2007) Connectivity-based parcellation of human cortex using diffusion MRI: Establishing reproducibility, validity and observer independence in BA 44/45 and SMA/pre-SMA. Neuroimage 34:204-211.

Koechlin E, Basso G, Pietrini P, Panzer S, Grafman J (1999) The role of the anterior prefrontal cortex in human cognition. Nature 399:148-151.

Konen CS, Kastner S (2008) Representation of eye movements and stimulus motion in topographically organized areas of human posterior parietal cortex. J Neurosci 28:8361-8375.

Koyama M, Hasegawa I, Osada T, Adachi Y, Nakahara K, Miyashita Y (2004) Functional magnetic resonance imaging of macaque monkeys perform- ing visually guided saccade tasks: comparison of cortical eye fields with humans. Neuron 41:795-807.

Krubitzer L (1995) The organization of neocortex in mammals: Are species differences really so different? Trends Neurosci 18:408-417.

Krubitzer L (2007) The magnificent compromise: Cortical field evolution in mammals. Neuron 56:201-208.

Levy I, Schluppeck D, Heeger DJ, Glimcher PW (2007) Specificity of human cortical areas for reaches and saccades. J Neurosci 27:4687-4696.

Lewis JW, Van Essen DC (2000) Corticocortical connections of visual, sensorimotor, and multimodal processing areas in the parietal lobe of the macaque monkey. J Comp Neurol 428:112-137.

Luppino G, Murata A, Govoni P, Matelli M (1999) Largely segregated parietofrontal connections linking rostral intraparietal cortex (areas AIP and VIP) and the ventral premotor cortex (areas F5 and F4). Exp Brain Res 128:181-187.

Margulies DS, Vincent JL, Kelly C, Lohmann G, Uddin LQ, Biswal BB, Villringer A, Castellanos FX, Milham MP, Petrides M (2009) Precuneus shares intrinsic functional architecture in humans and monkeys. Proc Natl Acad Sci U S A 106:20069-20074.

Mars RB, Coles MG, Hulstijn W, Toni I (2008) Delay-related cerebral activity and motor preparation. Cortex 44:507-520.

Matelli M, Camarda R, Glickstein M, Rizzolatti G (1986) Afferent and efferent projections of the inferior area 6 in the macaque monkey. J Comp Neurol 251:281-298.

Matelli M, Govoni P, Galletti C, Kutz DF, Luppino G (1998) Superior area 6 afferents from the superior parietal lobule in the macaque monkey. J Comp Neurol 402:327-352.

McLaren DG, Kosmatka KJ, Oakes TR, Kroenke CD, Kohama SG, Matochik JA, Ingram DK, Johnson SC (2009) A population-average MRI-based atlas collection of the rhesus macaque. Neuroimage 45:52-59.

Mort DJ, Perry RJ, Mannan SK, Hodgson TL, Anderson E, Quest R, McRobbie D, McBride A, Husain M, Kennard C (2003) Differential cortical activation during voluntary and reflexive saccades in man. Neuroimage 18:231-246.

Nelson SM, Cohen AL, Power JD, Wig GS, Miezin FM, Wheeler ME, Velanova K, Donaldson DI, Phillips JS, Schlaggar BL, Petersen SE (2010) A parcellation scheme for human left lateral parietal cortex. Neuron 67:156-170.

Orban GA, Claeys K, Nelissen K, Smans R, Sunaert S, Todd JT, Wardak C, Durand JB, Vanduffel W (2006) Mapping the parietal cortex of human and non-human primates. Neuropsychologia 44:2647-2667.

O’Reilly JX, Beckmann CF, Tomassini V, Ramnani N, Johansen-Berg H (2010) Distinct and overlapping functional zones in the cerebellum defined by resting state functional connectivity. Cereb Cortex 20:953-965.

Pandya DN, Yeterian EH (1996) Comparison of prefrontal architecture and connections. Philos Trans R Soc Lond B Biol Sci 351:1423-1432.

Passingham R (2009) How good is the macaque monkey model of the human brain? Curr Opin Neurobiol 19:6-11.

Passingham RE (2008) What is special about the human brain? Oxford; New York: Oxford University Press.

Passingham RE, Stephan KE, Kötter R (2002) The anatomical basis of functional localization in the cortex. Nat Rev Neurosci 3:606-616.

Patel GH, Shulman GL, Baker JT, Akbudak E, Snyder AZ, Snyder LH, Corbetta M (2010) Topographic organization of macaque area LIP. Proc Natl Acad Sci U S A 107:4728-4733.

Peeters R, Simone L, Nelissen K, Fabbri-Destro M, Vanduffel W, Rizzolatti G, Orban GA (2009) The representation of tool use in humans and monkeys: Common and uniquely human features. J Neurosci 29:11523-11539.

Petrides M, Pandya DN (2007) Efferent association pathways from the rostral prefrontal cortex in the macaque monkey. J Neurosci 27:11573-11586.

Rajkowska G, Goldman-Rakic PS (1995) Cytoarchitectonic definition of prefrontal areas in the normal human cortex: II. Variability in locations of areas 9 and 46 and relationship to the Talairach Coordinate System. Cereb Cortex 5:323-337.

Rozzi S, Calzavara R, Belmalih A, Borra E, Gregoriou GG, Matelli M, Luppino G (2006) Cortical connections of the inferior parietal cortical convexity of the macaque monkey. Cereb Cortex 16:1389-1417.

Rushworth MF, Paus T, Sipila PK (2001) Attention systems and the organization of the human parietal cortex. J Neurosci 21:5262-5271.

Rushworth MF, Behrens TE, Johansen-Berg H (2006) Connection patterns 
distinguish 3 regions of human parietal cortex. Cereb Cortex 16:14181430.

Rushworth MFS, Boorman E, Mars RB (2009) Comparing brain connections in different species using diffusion weighted imaging. In: Diffusion MRI. From quantitative measurement to in vivo neuroanatomy (Johansen-Berg H, Behrens TEJ, eds), pp445-460. Amsterdam: Academic Press.

Saleem KS, Logothetis NK (2006) A combinted MRI and histology atlas of the rhesus monkey brain. Amsterdam: Academic Press.

Scheperjans F, Hermann K, Eickhoff SB, Amunts K, Schleicher A, Zilles K (2008a) Observer-independent cytoarchitectonic mapping of the human superior parietal cortex. Cereb Cortex 18:846-867.

Scheperjans F, Eickhoff SB, Hömke L, Mohlberg H, Hermann K, Amunts K, Zilles K (2008b) Probabilistic maps, morphometry, and variability of cytoarchitectonic areas in the human superior parietal cortex. Cereb Cortex 18:2141-2157.

Schluppeck D, Glimcher P, Heeger DJ (2005) Topographic organization for delayed saccades in human posterior parietal cortex. J Neurophysiol 94:1372-1384.

Schluppeck D, Curtis CE, Glimcher PW, Heeger DJ (2006) Sustained activity in topographic areas of human posterior parietal cortex during memory-guided saccades. J Neurosci 26:5098-5108.

Schubotz RI, Anwander A, Knösche TR, von Cramon DY, Tittgemeyer M (2010) Anatomical and functional parcellation of the human lateral premotor cortex. Neuroimage 50:396-408.

Seltzer B, Pandya DN (1984) Further Observations on Parieto-Temporal Connections in the Rhesus-Monkey. Exp Brain Res 55:301-312.

Semendeferi K, Armstrong E, Schleicher A, Zilles K, Van Hoesen GW (2001) Prefrontal cortex in humans and apes: A comparative study of area 10. Am J Phys Anthropol 114:224-241.

Sereno MI, Huang RS (2006) A human parietal face area contains aligned head-centered visual and tactile maps. Nat Neurosci 9:1337-1343.

Sereno MI, Pitzalis S, Martinez A (2001) Mapping of contralateral space in retinotopic coordinates by a parietal cortical area in humans. Science 294:1350-1354.

Silver MA, Ress D, Heeger DJ (2005) Topographic maps of visual spatial attention in human parietal cortex. J Neurophysiol 94:1358-1371.

Simon O, Mangin JF, Cohen L, Le Bihan D, Dehaene S (2002) Topographical layout of hand, eye, calculation, and language-related areas in the human parietal lobe. Neuron 33:475-487.

Simon O, Kherif F, Flandin G, Poline JB, Rivière D, Mangin JF, Le Bihan D, Dehaene S (2004) Automatized clustering and functional geometry of human parietofrontal networks for language, space, and number. Neuroimage 23:1192-1202.

Smith SM, Jenkinson M, Woolrich MW, Beckmann CF, Behrens TE, Johansen-Berg H, Bannister PR, De Luca M, Drobnjak I, Flitney DE,
Niazy RK, Saunders J, Vickers J, Zhang Y, De Stefano N, Brady JM, Matthews PM (2004) Advances in functional and structural MR image analysis and implementation as FSL. Neuroimage 23 [Suppl 1]:S208-S219.

Snyder LH, Batista AP, Andersen RA (1997) Coding of intention in the posterior parietal cortex. Nature 386:167-170.

Suzuki M, Tsukiura T, Matsue Y, Yamadori A, Fujii T (2005) Dissociable brain activations during the retrieval of different kinds of spatial context memory. Neuroimage 25:993-1001.

Suzuki WA, Amaral DG (1994) Topographic organization of the reciprocal connections between the monkey entorhinal cortex and the perirhinal and parahippocampal cortices. J Neurosci 14:1856-1877.

Swisher JD, Halko MA, Merabet LB, McMains SA, Somers DC (2007) Visual topography of human intraparietal sulcus. J Neurosci 27:5326-5337.

Szczepanski SM, Konen CS, Kastner S (2010) Mechanisms of spatial attention control in frontal and parietal cortex. J Neurosci 30:148-160.

Tanné-Gariépy J, Rouiller EM, Boussaoud D (2002) Parietal inputs to dorsal versus ventral premotor areas in the macaque monkey: evidence for largely segregated visuomotor pathways. Exp Brain Res 145:91-103.

Thiel CM, Zilles K, Fink GR (2004) Cerebral correlates of alerting, orienting and reorienting of visuospatial attention: an event-related fMRI study. Neuroimage 21:318-328.

Tomassini V, Jbabdi S, Klein JC, Behrens TE, Pozzilli C, Matthews PM, Rushworth MF, Johansen-Berg H (2007) Diffusion-weighted imaging tractography-based parcellation of the human lateral premotor cortex identifies dorsal and ventral subregions with anatomical and functional specializations. J Neurosci 27:10259-10269.

Van Essen DC (2002) Windows on the brain. The emerging role of atlases and databases in neuroscience. Curr Opin Neurobiol 12:574-579.

Van Essen DC, Drury HA, Dickson J, Harwell J, Hanlon D, Anderson CH (2001) An integrated software system for surface-based analyses of cerebral cortex. J Am Med Inform Assoc 8:443-459.

Vanduffel W, Fize D, Peuskens H, Denys K, Sunaert S, Todd JT, Orban GA (2002a) Extracting 3D from motion: Differences in human and monkey intraparietal cortex. Science 298:413-415.

Vanduffel W, Tootell RB, Schoups AA, Orban GA (2002b) The organization of orientation selectivity throughout macaque visual cortex. Cereb Cortex 12:647-662.

Vincent JL, Patel GH, Fox MD, Snyder AZ, Baker JT, Van Essen DC, Zempel JM, Snyder LH, Corbetta M, Raichle ME (2007) Intrinsic functional architecture in the anaesthetized monkey brain. Nature 447:83-86.

Wise SP, Boussaoud D, Johnson PB, Caminiti R (1997) Premotor and parietal cortex: Corticocortical connectivity and combinatorial computations. Annu Rev Neurosci 20:25-42.

Xiang HD, Fonteijn HM, Norris DG, Hagoort P (2010) Topographical functional connectivity pattern in the perisylvian language networks. Cereb Cortex 20:549-560. 